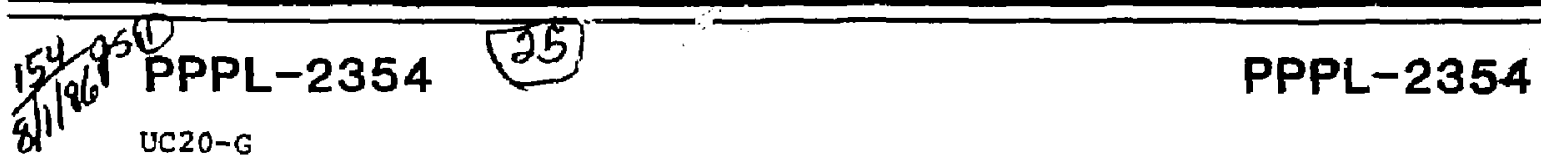

NONLINEAR EVOLUTION OF DRIFT INSTABILITIES IN THE PRESENCE OF COLLISIONS




This report was prepared as an account of work sponsored by the United states Government. Neither the United States nor the United States Department of Energy, nor any of thefr employees, nor any of their contractors, subcontractors, or their employees, makes any warranty, express or implied, or assumes any legal liablitity or responzibility for the accuracy, conpleteness or usefulness of any information, apparatus, product or process disclosed, or represents that its use wuld not infringe privately owmed rights.

Printed in the United States of America

Avallable from:

Vational Technical Information Service

U.5. Department of Commerce

5285 Port Róyal Road

Springfielt, Virginia 22161

Price Printed Copy $\$ \ldots \ldots$ _ Microfiche $\$ 4.50$

\section{- Pages}

$1=25$

25-50

51-75

76-100

101-125

126-150

151-175

176-200

201-225

226-250

251-275

276-300

$301-325$

326-350

351-375

$376-400$

$401-425$

426-450

451-475

476-500

$500-525$

526-550

$551-575$

567-600

NIIS

Selling Prige

$\$ 7.00$

$\$ 8.50$

$\$ 10.00$

$\$ 11.50$

$\$ 13.00$

$\$ 14.50$

$\$ 16.00$

$\$ 17.50$

$\$ 19.00$

$\$ 20.50$

$\$ 22.00$

$\$ 23.50$

$\$ 25.00$

$\$ 26.50$

$\$ 28.00$

$\$ 29.50$

$\$ 31.00$

$\$ 32.50$

$\$ 34.00$

$\$ 35.50$

$\$ 37.00$

$\$ 38.50$

$\$ 40.00$

$\$ 41.50$
For documents over 600 pages, add $\$ 1.50$ for each additional 25-page increment. 


\title{
Nonlinear Evolution of Drift Instabilities in the Presence of Collisions
}

\author{
J. F. Federici, W. W. Lee, and W. M. Tang \\ Plasma Physics Laboratory, Princeton University \\ Princeton, New Jersey 08544 \\ PPPE $-\mathbf{- 2 3 5 4}$ \\ DE86 013642
}

\begin{abstract}
' ABSTRACT
Nonlinear evolution of drift instabilities in the presence of electron-ion collisions in a shear-free slab has been studied by using gyrokinetic particle simulation techniques as well as by solving, both numerically and analy tically, model mode-coupling equat.jons. The purpose of the investigation is to determine the mechanisms responsible for the nonlinear saturation of the instability and for the ensuing steady-state transport. Such an insight is very valuable for understanding drift wave problems in more complicated geometries. The results indicate that the electron $\mathbf{E} \times \mathbf{B}$ convection is the dominant mechanism for saturation. It is also found that the saturation amplitude and the associated quasilinear diffusion are greatly enhanced over their collisionless values as a result of weak collisions. In the highly collisional (fluid) limit, there is an upper bound for saturation with $e \phi / T_{e} \simeq\left(\omega_{l} / \Omega_{i}\right) /\left(k_{\perp} \rho_{s}\right)^{2}$. The associated quasilinear diffusion, which increases with collisionality, takes the form of $D_{q l} \simeq \gamma_{l} / k_{\perp}^{2}$, where $\omega_{l}$ and $\gamma_{l}$ are the linear frequency and growth rate, respectively. In the steady state, the diffusion process becomes stochastic in nature. The relevant mechasisms here are related to the velocity-space nonlinearities and
\end{abstract}


background fluctuations. The magnitude of the diffusion at this stage can be comparzble to that of quasilinear diffision in the presence of collisions, and it remains finite even in the collisionless limit. 


\section{Introduction}

It is widely believed that drift-wave-related, small-scale, low-frequency fluctuations are the primary cause for the rapid cross-field transport observed in tokamaks. ${ }^{12}$ In recent years, substantial progress on the theoretical front has been made in understanding the characteristics of these fluctuations and their relation to confinement. ${ }^{2}$ One of the most important advances has been the identification of the nonlinear $\mathbf{E} \times \mathbf{B}$ convection of the jons as the key ingredient for the inverse energy cascade process which leads to broadly peaked frequency and wave number spectra in steady-state turbulence. ${ }^{3}$ This work has prompted a number of authors to address the issues of saturation and diffusion based on a fluid jon model for the instability in which the driving and damping mechanisms of the electrons have been incorporated in an ad hoc manner. ${ }^{4,5}$ Pursuing further along this line of research, Hasegawa and Wakatani ${ }^{6}$ have used a set of self-consistent fluid equations to study dissipative drift instabilities, while Walt $z^{7}$ has extended the approach to include additional physical effects pertinent to tokamaks. Terry and Diamond ${ }^{\mathbb{s}}$ have also studied the problems of collisional drift waves related to the edge turbulence in tokamaks using a combination of fluid and kinetic (ion Compton scattering) descriptions for the instability. So far, these attempts have helped us to gain considerable insight into the physical processes involved in microturbulence. However, there are several important questions with regard to the basic assumptions made in the aforementioned model equations which remain unanswered. First of all, is it indeed valid to exclude other nonlinear terms from the formalisn except for those due to the $\mathbf{E} \times \mathbf{B}$ convection? Moreover, are the physical processes and the parametric dependences at the saturation of the instability different from those in the steady state? Since most. of the present-day tokaniaks are operating at the collisionless to marginally collisional limit, it also brings up the question of whether $\mathrm{jt}$ is appropriate to describe 
microinstabilities in the edge region using fluid equations. These are basic issues which should be resolved before embarking upon the next phase of the rcsearch in nonlinear microturbulence.

In the present paper, we intend to provide some of the answers by using gyrokinetic particle simulation techniques ${ }^{9}$ as well as by solving, both numerically and analytically, the model mode-coupling equations for the investigation of the nonlinear evolution of drift instabilities in the presence of electron-ion collisions in a shear-free slab. There are several distinct advantages for adopting such an approach. The gyrophase-averaged Vlasov and Poisson eqquations ${ }^{9,10}$ used in this study contain all the nonlinearities relevant for describing low frequency phenomena; we can solve these equations in their entirety via particle simulation without making prior judgments concerning the relative importance of each of the nonlinear terms. In addition, by concentrating on a simple slab model, we carı readily identify various noulinear mechanisms responsible for saturation and transport in the simulation by comparing the results with the solutions from the nodel mode-coupling equations. Finally, unlike fluid codes, the versatility of a particle code enables us to investigate the instabilities in varjous colljsjonalities and gives us the necessary information for comparison with the experimental trends observed in the tokamak edge region, ${ }^{11-13}$ where the plasma is marginally collisional and drift instabilities are thought to be active.

The application of particle simulation to the study of drift waves has a long history. ${ }^{14}$ Due to the existence of high-frequency space-charge waves, the conventional codes, limited by large noise level as well as by small time steps and small grid sizes, are rather cumbersome for simulating low frequency plonomena with the characteristic length of the order of $\rho_{s}$, i.e., the ion gyroradjus measured at the electron temperature. The development of a gyrokinetic simulation scineme en- 
ables us to mitigate these difficulties. ${ }^{15,16}$ The gyrokinetic formalism also makes it possible to separate formally the spatial scales for the background inhomogeneity from those associated with the perturbations. ${ }^{15}$ Thus, we can study steady-state problems in the simulation without the dominating quasilinear profile modification processes. ${ }^{14}$ This new aspect of the simulation accurately reflects the realistic experimental situation, where the density and temperature gradients, which drive this class of instabilitjes, tend to persisi as quasi-static profiles due to the continuous replenishment of the plasma.

A two-dimensional (or pseudo-3D) gyrokinetic code has been used previously to investigate collisioniess drift instabilities in a snearless slab. ${ }^{17}$ It was found that the nonlinear $\mathbf{E} \times \mathbf{B}$ convection for the electrons gave rise to the saturation of the instability and that the electron velocity-space nonlinearitjes were related to the steady-state particle flux. These potentially important nonlinear mechanisms have generally been ignored in the cunfinement studies for tokamaks. The recent progress made in modelling microinstabilities in the edge region ${ }^{6,8}$ affords us an opportunity to assess the relevancy of these nonlinear interactions whet: the collisions are present. Thus, this paper may be considered as an extension of the work presented in Ref. 17.

In the present study, collisional effects were accounted for in the sinulation utilizing the Lorentz collision model. ${ }^{18} \mathrm{~A}$ one-dimensional diffusion model ${ }^{19}$ was used for the mode-coupling calculatjons. The results have indicated that the $\mathbf{E} \times \mathbf{B}$ convection of the electrons, which generates the nonljnear zero-frequency density response and, in turn, mullifies the phase difference between the linear density and potential fluctuations, is still the dominant mechanism for saturation regardless of collisions. The fluid aspect of this nonlinearity has been discussed in Refs. 20 and 21 , and its kinetic collisionless version has also been presented in Ref. 22.) It was also found that the saturation amplitude and the assocjated quasilinear diffusion were greatly en- 
hanced over their collisionless values as a result of weak collisions. This is somewhat unexpected since the liniear properties of these instabilities have not been affected by the collisions in any significant manner. In the highly collisional (fluid) limit, there is an upper bound for the saturation with $e \phi / T_{e} \simeq\left(\omega_{l} / \Omega_{i}\right) /\left(k_{\perp} \rho_{s}\right)^{2}$, where $\omega_{i}$ is the collision-independent linear mode frequency, $\rho_{s} \equiv C_{a} / \Omega_{i}, \Omega_{i} \equiv e B / m_{i} c$ and $C_{s} \equiv\left(T_{e} / m_{i}\right)^{\frac{1}{2}}$. This upper bound may be easily approached with a moder. ate collision rate. The associated quasilinear diffusion takes the well-known form of $D_{q l} \simeq \gamma_{l} / k_{\perp}^{2}$, where $\gamma_{l}$ is the linear growth rate and is linearly proportional to the collision rate. In the steady state, the diffusion process becomes stachastic in nature. The relevant mechanisms responsible for the outward particle flux are related to the velocity-space nonlinearities and the background fluctuations. The magnitude of the diffusion at this stage is again dependent on the collision rate and can be comparable to the corresponding quasilinear values when collisions are relatively strong, and it remains finite even in the absence of collisions. These are very interesting results since the electron dissipation seems to play a more vital sole in the monlinear stage of the instability thau what is commonly believed. Most significantly, our results are consistent with the trend observed in the actual experiments $^{11-13}$ which indicate that in the edge region, the fluct tation amplitude is rather insensitive to collisions whereas diffusion increases with density (i.є.. sensitive to collisions). We will elaborate on these points later.

The paper is organized as follows: the basic gyrokinetic formalism for studying drift instabilities in the presence of electron-ion collisions is given in Sec. II. Section III presents the gyrokinetic particle sinulation results, and Sec. IV describes the analytical and numerical calculations for the nodel mode-coupling equations. In Sec. V, a brief discussion and some concluding remarks are given. 


\section{Gyrokinetic Formalism and Simulation Techniques}

The gyropirase-averaged Vlasov and Poisson equations used in the present investigation have been derjved earlier ${ }^{9_{1} 10}$ based on the well-known gyrokinetic ordering. The ordering assumes that $\omega / \Omega, \rho / L, k_{\|} / k_{\perp}$ and $\epsilon \phi / T$ are of order $\epsilon$, where $\omega$ is the frequency of interest, $\Omega$ is the gyrofrequency, $\rho$ is the gyroradius, $L$ is the equilibrium scale length, $k_{\|}$and $k_{\perp}$ are the perturbed wave numbers parallel and perpendicular to the magnetic field, respertingl, ${ }^{*}$, is the electrostatic poten$t j a]$ normalized to he average plasma temperature, and $\epsilon$ is a smallness parameter. The gyrophaseraveraging procedure eliminates the fast gyromotion and, in turn, the gvrophase-dependent quantities from the system. Furthernore, to facilitate the steady-state simulation, the scale lengths for the background inhomogeneities can be separated from those associated with the perturbations by applying the multiple scale expansion scheme on the spatial derivatjve term in the reduced Vlasov equation.

The resulting gyrokinetic Vlasov equation in slab geometry valid to order $\epsilon^{2}$ can be written in terms of guiding-center variables $\left(\mathbf{R}, \mu, v_{\|}\right)$as $s^{9,10,15}$

$$
\frac{\partial F}{\partial t}+v_{\|} \tilde{\mathrm{b}} \cdot \frac{\partial F}{\partial \overline{\mathbf{R}}}-\frac{q}{m \Omega} \frac{\partial \Psi}{\partial \mathbf{R}} \times \hat{\mathbf{b}} \cdot\left(\frac{\partial F}{\partial \mathbf{R}}-\vec{x} F\right)-\frac{q}{m} \frac{\partial \Psi}{\partial \mathbf{R}} \cdot \hat{\mathrm{b}} \frac{\partial F}{\partial v_{\|}}=\mathbf{C}(F)
$$

where

$$
\Psi(\mathbf{R}) \equiv \bar{\phi}(\mathbf{R})-\frac{q}{2 T}\left(\frac{v_{t}}{\Omega}\right)^{2} \mid \frac{\partial \phi(\mathbf{R})}{\partial \mathbf{R}_{\perp}} i^{2}
$$

$F \equiv F\left(\mathbf{R}, \mu, v_{\|}, t\right)$ is the gyrophase-independen distribution function containing spatial perturbations only, $\vec{\kappa} \equiv-\nabla \ln n(\epsilon \mathbf{R})$ is the parameter for the background density gradient and is constant in time, $C(F)$ is the collision operator, $\mu \equiv$ $v_{-2}^{2} / 2, \Omega \equiv g B / m c, \overrightarrow{\mathrm{b}} \equiv \mathbf{B} / B, \mathbf{B}$ is the external maguetic field, $v_{t} \equiv(T / m)^{\frac{1}{2}}, \phi(\mathbf{R})$ and $\bar{\phi}(\mathbf{R})$ are the electrostatic potentials at the guiding center, and $q$ is the signed charge. Moreover, the guiding-center position variable $\mathbf{R}$ is related to the parti- 
"cle position variable $x$ through $R \equiv x-\vec{\rho}$, where $\vec{\rho} \equiv-v_{\perp} \times \vec{b} / \Omega$ is the particle gyroradius. Equation (1) js valid for both the electrons and the ions. However, for the electrons, the finite gyroradius effects are negligible and Eq. (1) reduces to the usual arift-kinetic equation for $\vec{p} \rightarrow 0$. The effects of electron-ion collisions cars be represented by the gyrophase-averaged Lorentz collision operator incluaing only pitch-angle scattering in the velocity-space for the electrons, ${ }^{\mathbf{2 3}, 24}$

$$
C\left(F_{e}\right) \equiv \frac{\nu_{e i}}{2 \sin \theta} \frac{\partial}{\partial \theta}\left[\sin \theta\left(\frac{\partial F_{e}}{\partial \theta}\right)\right]
$$

where $\nu_{e i} \equiv 4 \pi n_{e} e^{4} \ln \Lambda / m_{e}^{2} v_{t e}^{3}$ is the collision frequency and $\theta \equiv \cos ^{-1} v_{\|} /\left(v_{i}^{3}+v_{L}^{2}\right)^{\frac{1}{2}}$. Equation (3) conserves both number density and particle energy.

The gyrokinetic Poisson equation in particle coordinates takes the form ${ }^{9.10}$

$$
\nabla^{2} \phi-\frac{\tau(\dot{\varphi}-\bar{\phi})}{\lambda_{D}^{2}}+\left(\frac{\rho_{s}}{\lambda_{D}}\right)^{2} \nabla_{\perp} \cdot\left[\frac{\left(n_{i}-n_{o}\right)}{n_{o}} \nabla_{\perp} \phi\right]=-4 \pi e\left(\bar{n}_{i}-n_{e}\right),
$$

where the electrostatic potentials $\phi$ and $\bar{\phi}$, as well as the number densities, $\bar{n}_{i}, n_{i}$, and $n_{e}$, are functions of $\mathbf{x}, \nabla \equiv \partial / \partial x, \lambda_{D}^{2} \equiv T_{e} / 4 \pi n_{o} \epsilon^{2}, \tau \equiv T_{e} / T_{i}, \rho_{s} \equiv \sqrt{\tau} \rho_{i}, \rho_{i} \equiv$ $\left(T_{i} / m_{i}\right)^{\frac{1}{2}} / \Omega_{i}$, and subscripts $e$ and $\mathrm{i}$ denote species. The relationslips between the distribution fumction, the potentials and the number densities in Eqs. (1), (2), and (4) are as follows. Ir terms of Fourier transiorms with

$$
\phi(\mathbf{x})=\sum_{\mathbf{k}} \phi(\mathbf{k}) \exp (\mathbf{i k} \cdot \mathbf{x})
$$

and

$$
F(\mathbf{R})=\sum_{\mathbf{k}} F(\mathbf{k}) \exp (i \mathbf{k} \cdot \mathbf{R})
$$

the coordinate-transformed quantities can be expressed as

$$
\begin{aligned}
\bar{\phi}(\mathbf{R}) & =\sum_{\mathbf{k}} \phi(\mathbf{k}) J_{a}\left(\frac{k_{\perp} v_{\perp}}{\Omega}\right) \exp (i \mathbf{k} \cdot \mathbf{R}) \\
\bar{n}(\mathbf{x}) & =\sum_{\mathbf{k}} \int F(\mathbf{k}) J_{o}\left(\frac{k_{\perp} v_{-}}{\Omega}\right) \exp (i \mathbf{k} \cdot \mathbf{x}) d \mu d v_{i}, \\
\bar{\phi}(\mathbf{x}) & =\sum_{\mathbf{k}} \phi(\mathbf{k}) \Gamma_{o}(b) \operatorname{eip}(i \mathbf{k} \cdot \mathbf{x})
\end{aligned}
$$




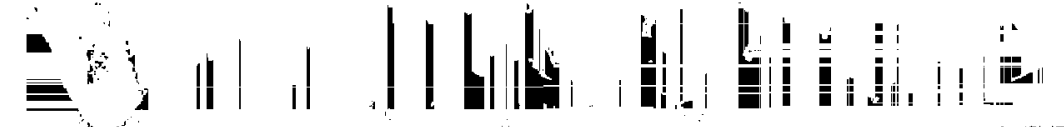

where the ordinary bessel function $J_{0} \equiv\langle\exp (i k \cdot \rho)\rangle_{\infty}$ is the result of the gyrophase avoraging prccess, $\Gamma_{\mathrm{o}}(b) \equiv I_{o}(b) \exp (-b)$ cumes from the second gyrophase averaging with respect to the Max elliati background, $b \equiv k_{\perp}^{2} \rho_{i}^{2}, I_{a}$ is the madified Bessel function, $\phi(\mathbf{R})$ and $n(\mathbf{x})$ correspond, respectively, to $\bar{\phi}(\mathbf{R})$ and $\bar{n}(\mathbf{x})$ with $J_{0}=1$, and $n_{0} \equiv\langle n(x)\rangle_{x}$ is the spatially averaged number density. In Eq. (4), $n_{e}=0$ is assumed, and the additione! terms on the left-hand side of the equation are the contributions from the ion polarization density response. For $b \ll 1$, the lowest order term becomes $\left(\rho_{*} / \lambda_{D}\right)^{2} \nabla_{-}^{2} \phi$. Since $\rho_{A} \gg \lambda_{D}$ for the usual tokamak plasmas, the Delye shielding term of $\nabla^{2} \phi$ is mucl stnaller by comparison and, therefore, can be ignored in Eq. (4). Finally, in Eqs. (2) and (4) we have kept only the leading t.erms from the small gyroradius expajssion for $\eta\left(\varepsilon^{2}\right)$ quantities.

Equations (1) and (4) have been solved numerically in the present investigation using particle simulation techniques as described in Ref. 1.5. The techniques are similar to the standard particle pushing schemes. The only complication comes from the term associated with the background inhomogeneity $\vec{R}$ in $\mathrm{Eq}$. (1) which gives rise to a new equation of motion for the particles. The advamage of isolating $n$. and keeping it constant in time is to enable us to carry out simulations for steadystate turbulence without the interference of quasilinear profile modification. In order to save comprtational time, an efficient numerical schene to expedite the coordinate transformation of Eqs. (5) and (6) has also been devised.

The Eorentz operator, Eq. (3), has been evaluated with the help of the numerical sclseme presented in Ref. 18. At each time step, the scattering angle in the velocity space for the $\mathrm{j}$-th electron against the stationary ion background is given by

$$
\Delta \theta_{j}=\left[-2 \nu_{e i} \Delta t \ln \left(1-r_{j}\right)^{\frac{1}{2}}\right.
$$

where $\Delta t$ is the time step for the simulation and $r$ is a random number between 
$(0,1)$. The new velocities then become

$$
\begin{aligned}
& v_{\| j}^{\prime}=v_{\| j}\left[1-\left(\Delta \theta_{j}\right)^{2}\right]^{\frac{1}{2}}-v_{\perp}\left(\Delta \theta_{j}\right) \sin \varphi_{j}, \\
& v_{\perp j}^{\prime}=\left[v_{\| j}^{2}+v_{\perp j}^{2}-v_{\| j}^{\prime}\right]^{\frac{1}{2}},
\end{aligned}
$$

where $\varphi$ is a random number between $(0,2 \pi)$. There are more efficient numerical methods to evaluate the Lorentz operator. ${ }^{25}$ However, we prefer the present scheme because of its accuracy.

\section{Gyrokinetic Particle Simulation Results}

Before we present the simulation results, let us discuss briefly the effect of collisions on the linear stability of drift waves in a shearless slab using the simple number-conserving $\mathrm{BGK}$ operator, ${ }^{26}$

$$
C\left(f_{e}\right) \equiv-\nu_{e i}\left(f_{e}-F_{M e} \int f_{e} d \mu d v_{||}\right)
$$

where $F_{s} \equiv F_{y_{c}}-f_{\mathrm{e}}$. The linear dispersion relation can be easily derived from Eqs. (1) and (4) and it lakes the familiar form of

$$
\frac{1+\left(1-\omega_{-} / \bar{\omega}\right) X_{e}}{J+i X_{e} \nu_{r} / \bar{\omega}}+\tau+\left(\tau \div \frac{\omega_{-}}{\omega}\right) \Gamma_{\mathrm{o}} X_{i}=0
$$

where $\omega$ is the normal mode freg̨uency. $\omega_{.} \equiv k_{y} \rho_{s} \kappa \rho_{s} \Omega_{i}$ is the diamagnetic drift frequency, $\bar{\omega} \equiv \omega+i \nu_{e} . X_{e} \equiv \xi_{e} \mathcal{Z}\left(\xi_{e}\right), \xi_{e} \equiv \tilde{\omega} / \sqrt{2} k_{\|} v_{t e}, X_{i} \equiv \xi_{i} Z\left(\xi_{i}\right), \xi_{i} \equiv \omega / \sqrt{2} k_{\|} v_{t i}, \tau \equiv$ $T_{\epsilon} / T_{i}, \Gamma_{o} \equiv I_{o}(b) \exp (-b)$, and $b \equiv\left(k_{x}^{2}+k_{y}^{2}\right) p_{i}^{2}$. For $\nu_{e i}=0$, Eq. (12) reduces to the usual collisionless result. In this limit, for $\left|\xi_{\mathrm{e}}\right| \ll 1,\left|\xi_{i}\right| \gg 1$, and $\Gamma \simeq 1-b$, the linear mode frequency and growth rate are given by

$$
\frac{\omega_{1}}{\omega_{.}}=\frac{1-b}{1+\tau b}
$$

and 


$$
\frac{\gamma_{l}}{\omega_{l}}=\frac{1}{1+\tau b} \sqrt{\frac{\pi}{2}} \frac{\omega_{-}-\omega_{l}}{k_{\|} v_{t e}},
$$

respectively. On the other hand, in the strongly collisional limit, where $\nu_{e i} / k_{\|} v_{t e} \gg$ 1, hence, $\left|\xi_{\varepsilon}\right| \gg 1$, solutions from Eq.(12) indicate that the linear mode frequency remains unaffected by collisions, i.e., it is still given by Eq. (13), and the growth rate is increased to

$$
\frac{\gamma_{l}}{\omega_{l}}=\frac{1}{1+\tau b} \frac{\nu_{e i}}{k_{\|} v_{\mathrm{ce}}} \frac{\omega_{-}-\omega_{l}}{k_{l \mid} v_{t e}},
$$

From Eqs. (13)-(15), one can see that the linear properties for weakly collisional as weil as marginally collisional $\left(\nu_{e i} / k_{\mathrm{ij}} \mathrm{v}_{t e} \simeq 1\right.$ ) drift waves do not deviate significantly from their collisionless values. The maximum growth rete for both limits occurs at $\tau b \simeq \Omega \tau /(3+\tau)$. It should be pointed out here that the Lorentz collision operator, Eq. (3), also gives the same frequency and growth rate for colljsion-dominated drift waves as those obtained from the BGK operator, Eq. (11). This can be easily understood by keeping in mind that the electrons are treated as a cold fluid in this limit and that both operators are number conserving and yjeld the same velocity moment.

$$
\int v_{\|} C\left(f_{\epsilon}\right) d \mu d v_{\|}=-v_{\mathrm{e} i} \int v_{\|} f_{\mathrm{e}} d \mu d v_{\|}
$$

Conrequently, they lead to the same dispersion relation. The linear properties described here are important for deternining the proper parameters for the simulation.

The simulation has been carried out with a two-dinensional $\left(x, y, v_{\|}, v_{\perp}\right)$ gyrokinetic code in slab geometry. The constant zerath-arder density inhomogeneity is in the $\mathrm{x}$ direction, j.e., $\vec{k} \equiv \kappa \hat{\mathbf{x}}$, and the external magnetic field, $\mathbf{B} \equiv \boldsymbol{B}_{\mathrm{o}}\left(\hat{\mathbf{z}}+\theta_{B} \hat{\mathbf{y}}\right)$, is in the $y-z$ plane, where $\theta_{B}$ is a small constant. Since this is a shear-free system, we can assume that both the particles and the waves are periodic in both the $x$ and $y$ directions. In terms of grid size $\Delta$ and ion cyclotron frequency $\Omega_{i}$, the sinulation parameters are: $L_{* x} \times L_{y}=32 \Delta \times 32 \Delta, n_{o}=16 / \Delta^{2}, m_{i} / m_{e}=183 \pi, T_{c} / T_{i}=4$, particle size $/ \Delta=2, \theta_{B}=k_{\|} / k_{\perp}=(0.002,0.01), \rho_{s} / \Delta=4.286, \kappa \rho_{s}=0.214,\left(k_{x} \rho_{s}, k_{y} \rho_{s}\right)=$ 
$(0.842 m, 0.842 n), \omega_{.} / \Omega_{i}=0.18 n$, where $(m, n)=0, \pm 1, \pm 2, \ldots, \nu_{e i} / \Omega_{i}=(0,0.001$, $0.01), \Omega_{i} \Delta t=1.09$, and $\Delta t$ is the time siep used in the code. The electrostatic potentials for $k_{z}=0$ modes have been set to zero, i.e., $\phi(m=0, n \neq 0)=0$, so as to elininate the one-dimensional (local) modes from the simulation and to satisfy the requirement of $\left|k_{x}\right| \gg|\kappa|$ imposed by the multiple spatial scale expansion. ${ }^{15}$ In order to simplify the nonlinear physics involved, some of the ambipolar potentials, $\phi(m \neq 0, n=0)$, have been selectively suppressed in the code as well. We will explain this later.

In the collisionless limit, numerical solutions of the dispersion relation, Eq. (12), indicate that only a bandful of the modes in the system are unstable. and the most unstable $(m=1 . n= \pm 1)$ modes have $\omega / \Omega_{i}= \pm 0.06+0.011 i$ for $t .3=0.01$ and $\omega / \Omega_{\mathrm{i}}= \pm 0.034+0.02 \pi i$ for $\theta_{B}=0.002$. [Egs. (13) and (14), which give slightly different values for these modes, concur with the fact that they are indeed the most. unstable ones.] Thus, the longest wavelength modes in the system also have the largest growth rates. This particular choice of parameters is intended to avoid the inverse energy cascade so that the resulting steady-state fluctuation spectrun can be nearly colierent. ${ }^{17}$ The purpose here is to facilitate the analytical understanding of the simulation results. For the collision rates used in the simulation, we do not expect the linear properties of the instability to deviate much from their collisionless counterparts. However, as we will discuss later, the noninear behavior of the instability depends critically on the collisionality, even for $\nu_{e i} k_{\| !} v_{t e} \& 1$. The simulation results for the two cases of $\theta_{B}$ are as follows.

\section{A. Case 1: $\theta_{B}=0.01$}

The collisionless limit of this case has been studied in Ref. 17. It was a rather weak instability, where the saturation amplitude for the nost unstable modes in the 
system, i.e., ( $m=1, n= \pm 1)$ and their complex conjugates, did not go much beyond the numerical noise level of $e \phi / T_{e} \simeq 0.65 \%$. However, with the introduction of collisions, the saturatjon ampiitude greatly increases. The time evolution of $\phi(1,1)$ for $\nu_{\mathrm{c} i} / \Omega_{i}=0.01$ is shown in Fig. 1, where the instability clearly exhibits two distinct stages of development, i.e., the linear growth stage and the nonlinear oscillations associated with saturation. The measured linear mode frequency and growtb rate are $\omega_{i} / \Omega_{i}=0.06$ and $\gamma_{i} / \Omega_{i}=0.013$, respectively, and the mode frequency after the saturation is about 0.05 . All of these numbers are very close to the collisionless linear values quoted earlier. The saturation occurs at $e \phi / T_{\varepsilon} \approx 10 \%$ and, in the steady state of the evolution, the average amplitude stays around $8 \%$. In Fig. 1, we have also indicated the average saturation amplit ude for $\nu_{\varepsilon i} / \Omega_{i}=0.0$ and 0.001 . They are $0.8 \%$ and $3 \%$, respectively. Thus, as we can see, relatively weak collisions $\left(\nu_{i i} / h_{\|} v_{t e}=0,0.003,0.03\right)$ have an enormous impact on the nonlinear behavjor of the instability. In fact, as we will demonstrate later, the nonlinear evolution for $\nu_{e} / \Omega_{1}=0.01$ closely resembles to that of the dissipative (collision-dominated) drift instabilities. In addition to $\phi(1,1)$ modes, $\phi(1,-1)$ modes have also been observed to grow from the discrete particle noise to a similar level of saturation (at somewhat different times) with the similar characteristics. (A detailed discussion has been given in Ref. 17.] As for the accompanying density fluctuations, the dominant modes are $(1, \pm 1),(2,0)$, and $(0,2)$. The fuctuation amplitudes in the steady state are $\delta n_{e}(1,1) / n_{0} \simeq i \%$ and $\delta n_{i}(1,1) / n_{0} \simeq 15 \%$ for $\nu_{e i} / \Omega_{i}=0.01$. The $(2,0)$ and $(0,2)$ modes are generated by the nonlinear effects and are responsible for saturation and transport, on which we will elaborate. In the simulation, we have also included $\phi(2,0)$ modes, which gave us better energy conservation. However, their absence did not alter the gross behavior of the instability. All the other modes of the system are much smaller in amplitude and remained at or slightly above the noise level throughout the simulation. 
As indicated in Ref. 17, the determination of anomalous particle diffusion due to drift instrabilities has been greatly facilitated because of the use of multiple spatial scale expansion in the simulation. Since the background inhomogeneity is constant as a function of time, the entire simulation volume can be viewed as a small segment of the density profile. As such, diffusion coefficients calculated from the global averages of the simulation are also the local values for the much larger system. Thus, following the arguments made in Ref. 17 , one can then evaluate the local diffusion coefficjent by

$$
D=\frac{\left\langle\Gamma_{\mathrm{ex}}\right\rangle}{\kappa}
$$

where $r$ is the inverse density scale length,

$$
\left\langle\Gamma_{e x}\right\rangle=\sum_{j=1}^{N} \frac{v_{e x}\left(x_{j}\right)}{N}
$$

is the spatially averaged electron particle flux in the inhomogeneous ( $x$ ) direction, $\mathbf{x}_{j}$ is the position of the $\mathrm{j}$-th particle, $v_{e x} \equiv-(c / B)(\partial \phi / \partial y)$ is the particle $\mathbf{E} \times \mathrm{B}$ drift, and $\mathrm{N}$ is the total number of electrons in the simulation. Because of the periodic boundary conditions imposed for the waves, the particle diffusion in our system is ambipolar, i.e. $\left\langle\Gamma_{e x}\right\rangle=\left\langle\Gamma_{i x}\right\rangle$, which can be verified by using Eqs. (1), (2), and (4), and by keeping in mind that $v_{i x} \equiv-(c / B)(\partial \Psi / O \mathbf{R}) \times \hat{\mathbf{b}} \cdot \hat{\mathbf{x}} .^{15}$

The diffusion coefficient. D (in units of $\rho_{s}^{2} \Omega_{1}$ ) as a function of time due to the instability for $\nu_{e i} / \Omega_{i}=0.01$ is shown in Fig. 2, where a bandpass filter of the width of $\omega$ has been applied. The first peak at $\Omega_{i} t=550$, which coincides with the saturation of the instability, gives the quasilinear diffusion coefficient, $D_{q i}=$ 0.0077 . After a rather prolonged period of relaxation, it settles to a lower steadystate value of $D=0.0038$. It is interesting to observe here that there seems to be a correlation between $t$ je oscillations of $D$ and $\left|e \phi / T_{e}\right|^{2}$ in Fig. 1. Figure 2 also includes the steady-state coefficients of $\mathrm{D}=0.0002$ and 0.001 for $\nu_{e i} / \Omega_{i}=0$ and 0.001 , respectively. Again, the dramatic increase in particle diffusion due to 
collisjons is evident. However, these numbers are significantly smaller than the Bohm coefficient, which is 0.0625 in our units.

\section{B. Case 2: $\theta_{B}=0.002$}

This case in its collisionless Jimit has also been investigated in Ref. 17. It was a much stronger instability, in which the dominant potentials are the $(1, \pm 1)$ modes and the dominant density fluctuations are the $(1, \pm 1),(2,0)$, and $(0,2)$ modes. As reported earlier, the instability reached an amplitude of $e \phi / T_{e}=8 \%$ at saturation before settling to the steady-state value of $4.4 \%$. Here, we have found that, although collisions also increase the saturation amplitude, they do not have the same impact as in the previous case. Figure 3 shows the time evolution of $\phi(1,1)$ for $\nu_{e i} / \Omega_{i}=$ 0.001. The measured linear made frequency and growth rate follow closely the theoretically predicted collisionless values. In the steady state, the mode frequency increases slightly to $\omega / \Omega_{i}=0.04$. The results in Fig. 3 also indicate that tine potentia! attains a maximum anplitude of $\epsilon \phi / T_{\epsilon}=9 \%$ at saturation and then reduces to $6 \%$ in the steady state. For $\nu_{e i} / \Omega_{i}=0.01$, the saturation amplitude and the steady-state amplitude are $12 \%$ ar:d $11 \%$, respectively. The corresponding density fluct.uations are $\delta n_{e}(1,1) / n_{0} \simeq 20 \%$ and $\delta n_{i}(1,1) / n_{0} \simeq 30 \%$. The amplitude of the potential in the steady state and that of the collisionless case are also indicated in Fig. 3. As in Case 1, we have also included the $\phi(2,0)$ mode in the simulation for the purpose of satisfying better energy conservation. It has no significant effect. on saturation and transport.

The particle diffusion for $\nu_{e i} / \Omega_{i}=0.001$ is shown in Fig. 4, where a bandpass filter of the width $\omega$ has been applied. The quasilinear peak occurs at $\Omega_{7} t=200$ with $D_{q l}=0.025$ (the unfiltered value is 0.035 ), and, after a long relaxation period, the steady-state diffusion coefficient achieves $\mathrm{D}=0.01$. The corresponding values 
for $\nu_{e i} / \Omega_{i}=0$ and 0.01 are given in Fig. 4 as well. They are $D=0.0025$ and 0.025 , respectively. (The steady-state value for the collisionless case is lower than the one quoted in Ref. 17, in which the average was taken for the time period of $\Omega_{\tau} t^{t}=250-650$.) As we can see, collisions again have an encrmous effect on the particle transport. Even for the modest collision rates used here $\left\{\nu_{t i} / k_{i f} v_{t e}=\right.$ $0,0.014,0.14)$, there is an order of magnitude difference in diffusion, and the largest $\mathrm{D}$ is comparable to the Bohm value. Another important aspect of the simulation is that particle diffusion remains finite even in the absence of collisions. This brings up the question of intrinsic (numerical) collision in the simulation. Its magnitude can be heuristically determined from the estimates for unmagnetized plasmas ${ }^{2 \pi}$ as follows. Since $\omega_{H}\left[\equiv\left(k_{\|} / k_{i}\right) \sqrt{m_{i} / m_{e}} \Omega_{i}\right]$ plays the role of $\omega_{\text {pe }}$ in the gyrokinetic plasma, ${ }^{15}$ the numerical relaxation (collision) time can be written as $\omega_{H} T_{c} \simeq 2 \pi N_{0}$, where $N_{0}$ is the number of particles in one wavelength, $2 \pi / k$, for the two-dimensional waves in the simulation. For the $(1, \pm 1)$ modes, $\Omega_{i} \tau_{c}$ is about three orders of magnitude larger than our simulation time. This estimate is consistent with our experience with the code. Thus, the simulation plasna is indeed intrinsically collisionless.

In the next two sections, we will present the interpretation of the simulation results and their relationship with the data from actual experiments. In our opinion, it is vitally important to understand such behavior before proceeding to investigate microjustability problems in more complicated geometries.

\section{Mode-Coupling Calculations}

The purpose of the following analytic and numerical analyses of model modecoupling equations is to understand the results described in Sec. III concerning the nonliuear behavior of drift instabilities in the presence of collisions, and, in particular, to identify the mechanisms responsible for the observed saturation and 
diffusion. The reduced mode-coupling equations in $\mathbf{k}$-space are obtained from the gyrokinetic Vlasov and Poisson equations, Eqs. (1) and (4), by keeping only the modes which are dominant in the simulation. To further simplify the calculation, we also assume that the distribution function $F\left(\mathbf{R}, \mu, v_{\|}, t\right)$ remains Maxwellian in $v_{\perp}$ at all times. This is certainly not exactly true for our simulation, where $F$ deviates from Maxwellian for the ejectrons through pitch angle scattering, and for the ions, the nonlinear interactions associated with $\rho_{i} \neq 0$, thus, $J_{o} \neq 0$ modify $F$. Nevertheless, the approach adopted here will enable us to ascertain the validity of such an approximation as well as the effect of the non-Maxwellian feature in $F$ on saturation and transport by comparing the sinulation and mode-coupling results.

To obtain model mode-coupling equations in $\left(\mathbf{k}, v_{\|}, t\right)$ space from Eqs. (1) to (7) we assume $F=n_{o}\left(1 / v_{t e}^{2}\right) \exp \left(-\mu / v_{t e}^{2}\right)\left(F_{M}+f\right)$ and perform the integration in $\mu$ space on Eqs. (1) and (3), where $F_{M}$ is the zeroth-order Maxwellian distribution in $v_{\|}$, i.e., $F_{M} \equiv\left(1 / \sqrt{2 \pi} v_{t e}\right) \exp \left(-v_{\|}^{2} / 2 v_{t e}^{2}\right)$. As in Ref. 17 , the resulting gyrokinetic Vlasov and Poisson equations in $\mathbf{k}$ space are then expressed in units of $\Omega_{i}^{-1}, \rho_{s}, e / T_{e}$ and $n_{o}$ for time, length, potential and number density, respectively. In terms of the dominant $(1, \pm 1),(2,0)$, and $(0,2)$ modes, denoted by, \pm 0 and 2 , respect.ively, we arrive at

$$
\begin{aligned}
& \frac{\partial f_{+}^{e}}{\partial t}+i k_{\|} v_{\|} f_{+}^{e}+i\left(\omega_{=}-k_{i \mid} u_{\|}\right) \phi_{+} F_{M}^{e} \\
& +\left[k_{\perp}^{2}+i v_{t e}^{2} k_{\|} \frac{\partial}{\partial v_{\|}}\right]\left(\phi_{-}^{*} f_{o}^{e}-\phi_{-} f_{2}^{e}\right)=C\left(f_{+}^{e}\right), \\
& \frac{\partial f_{-}^{e}}{\partial t}-i k_{\|\|} v_{\|} f_{-}^{e}-i\left(\omega_{*}-k_{i \mid} v_{\|}\right) \phi_{-} F_{M}^{e} \\
& -\left[k_{\perp}^{2}+i v_{t e}^{2} k_{\|} \frac{\partial}{\partial v_{\mu}}\right]\left(\dot{\phi}_{+}^{*} f_{o}^{\epsilon}-\phi_{+} f_{2}^{e *}\right)=C\left(f_{-}^{e}\right), \\
& \frac{\partial f_{o}^{*}}{\partial t}+\left[k_{\perp}^{2}+i v_{t e}^{2} k_{\|} \frac{\partial}{\partial v_{\|}}\right]\left(\phi_{+} f_{-}^{e}-\phi_{-} f_{+}^{e}\right)=C\left(f_{o}^{e}\right),
\end{aligned}
$$




$$
\begin{aligned}
& \frac{\partial f_{2}^{e}}{\partial t}+2 i k_{\|} v_{\|} f_{2}^{e}+k_{\perp}^{2}\left(\phi_{-}^{*} f_{+}^{e}-\phi_{+} f_{-}^{c *}\right) \\
& +i v_{t e}^{2} k_{\|} \frac{\partial}{\partial v_{\|}}\left(\phi_{-}^{*} f_{+}^{e}+\phi_{+} f_{-}^{e *}\right)=C\left(f_{2}^{e}\right)
\end{aligned}
$$

for the electrons, where $k_{\perp}^{2} \equiv \tau b \equiv k_{x}^{2}+k_{y}^{2}=2 k_{x} k_{y}, k_{y} k_{x}$ are the wave numbers for the $(1,1)$ mode, $\omega . \equiv k_{y} r_{i}, v_{t e}^{2} \equiv m_{i} / m_{\mathrm{e}}$, and

$$
C\left(f^{e}\right) \equiv \nu_{e i} \frac{\partial}{\partial v_{\|}}\left[v_{t e}^{2} \frac{\partial f^{e}}{\partial v_{\|}}+v_{\|} f^{e}\right]
$$

is the one-dimensional diffusion model collision operator. ${ }^{19}$ Equation (23), which is a reduced form of $\mathrm{Eq}$. (3) for the case when the particle distribution is Maxwellian in $v_{\perp}$, conserves number density, but not energy, and yields the same velocity moment as Eq. (16). The mode-coupling equations for the ions can be obtained by making the following replacements in Eqs. $(19)-(22): f^{e} \rightarrow f^{i}, v_{t e}^{2} \rightarrow-1, \nu_{e i} \rightarrow 0$, and $\pm i\left(\omega_{*}-k_{\|} v_{\|}\right) \phi_{ \pm} F_{M}^{e}- \pm i\left(\omega_{*}+\tau k_{\|} v_{\|}\right) \phi_{ \pm} F_{M}^{i}(1-b)$. All the potentials in Eqs. (21) and (22) should also be modified by a factor of (1-b). The resulting Poisson equations for $k^{2} \lambda_{D}^{2} \ll 1$ and $b \ll 1$ become

$$
\begin{aligned}
k_{\perp}^{2} \phi_{ \pm} & =n_{ \pm}^{i}-n_{=}^{\epsilon}, \\
n_{2}^{i} & =n_{2}^{e},
\end{aligned}
$$

where

$$
n=\int f d v_{\|}
$$

and the $\vartheta\left(\epsilon^{2}\right)$ terms in $\mathrm{Eq}$. (25) are ignored. In accordance with our simulation model, we have set $\phi_{0}=\phi_{2}=0$ in the formulation. As a result, there is a problem of redundancy concerning Eq. (25), which we will discuss. This system of equations describes a general case of four-mode coupling.

The energy balance of the system is

$$
k_{\perp}^{2} \frac{d}{d t}\left(\left|\phi_{+}\right|^{2}+\left|\phi_{-}\right|^{2}\right)=2 k_{\|} \operatorname{Im}\left(\phi_{-}^{*} \int v_{\|} f_{-}^{e} d v_{\|}-\phi_{+}^{*} \int v_{\|} f_{+}^{e} d v_{\|}\right)
$$


where the current contribution from the ions is neglected. This result leads to

$$
\frac{d}{d t}\left[k_{\perp}^{2}\left(\left|\phi_{-}\right|^{2}+\left|\phi_{-}\right|^{2}\right)+\frac{1}{2 v_{t e}^{2}} \int v_{\|}^{2} f_{\infty}^{e} d v_{\|}\right]=-\frac{\nu_{e i}}{v_{t e}^{n}} \int v_{\|}^{2} f_{\infty}^{e} d v_{\|},
$$

where $f_{\infty}^{e}$ is the perturbed distribution for $\mathbf{k}=0$. Thus, the energy for aur simplified system is not conserved because of the particular collision model we have chosen. However, this is not really a cause for concern. Since $f_{\infty}^{c}$ is expected to have minimal effect on the nonlinear behavior of the instability, it is not included in Eqs. (19)-(22). Therefore, Eq. (27) is sufficient for our purpose.

For analytic and numerical reasons, it is sometimes advantageous for us to solve the mode-coupling equations in the Fourier-transformed velocity space. Letting

$$
\bar{f}^{e}(q)=\int_{-\varepsilon=}^{\infty} f^{c}\left(v_{\|}\right) \exp \left[i q v_{i !}\right] d v_{l i},
$$

Eqs. (19)-(22) then become

$$
\begin{aligned}
& {\left[\frac{\partial}{\partial \phi}+\left(k_{\|}+q \nu_{e i}\right) \frac{\partial}{\partial q}+q^{2} v_{t e}^{2} \nu_{e i}\right] \overline{f_{+}^{e}}(q)+\left[i \omega_{+}+k_{\mid i} q v_{t e}^{2}\right] \phi_{+} \overline{F_{M}}} \\
& \left.+\left[k_{\perp}^{2}+k_{\| \mid q v_{t r}^{2}}^{2}\right] \mid \phi_{-}^{*} \overline{f_{0}^{e}}(q)-a_{-} \overline{f_{!}^{e}}(q)\right\}=0 \\
& {\left[\frac{\partial}{\partial t}-\left(k_{\|}-q \nu_{e i}\right) \frac{\partial}{\partial q}+q^{2} v_{t e}^{2} \nu_{e i}\right] \overline{f_{-}^{e}}(q)-\left[i \omega_{*}+k_{\| \eta} q v_{t e}^{2}\right] \phi_{-} \overline{F_{M}^{e}}} \\
& -\left[k_{\perp}^{2}+k_{\|} q v_{\mathrm{te}}^{2}\right]\left[\phi_{+}^{*} \overline{f_{0}^{e}}(q)-\phi_{+} \overline{f_{2}^{*}}(-q)\right]=0 \\
& {\left[\frac{\partial}{\partial t}+q \nu_{e i} \frac{\partial}{\partial q}+q^{2} v_{t e}^{2} \nu_{e i}\right] \overline{f_{o}^{e}}(q)+\left[k_{\perp}^{2}+k_{\|} \mid q v_{t e}^{2}\right]\left[\phi_{+} \overline{f_{-}^{e}}(q)-\phi_{-} \overline{f_{+}^{e}}(q)\right]=0} \\
& {\left[\frac{\partial}{\partial t}+\left(2 k_{\|}+q \nu_{e i}\right) \frac{\partial}{\partial q}+q^{2} v_{t e}^{2} \nu_{e i}\right] \overline{f_{2}^{e}}(q)} \\
& +k_{\perp}^{2}\left[\phi_{-}^{*} \overline{f_{+}^{e}}(q)-\phi_{+} \overline{f_{-}^{e *}}(-q)\right]+k_{\|} q v_{L_{q}}^{2}\left[\phi_{-}^{*} \overline{f_{+}^{e}}(q)+\phi_{+} \overline{f_{-}^{e *}}(-q)\right]=0,
\end{aligned}
$$

where $\overline{F_{W}^{g}} \equiv \exp \left[-q^{2} v_{t e}^{2} / 2\right]$. To obtain the jon mode-coupling equations, one simply uses the following replacements: $\overline{f^{e}} \rightarrow \overline{f^{i}}, v_{t e}^{2} \rightarrow-1, \nu_{\mathrm{ei}} \rightarrow 0$, and $\pm\left[i \omega_{.}+\right.$ 
$\left.k_{\|} q v_{t e}^{2}\right] \phi_{ \pm} \overline{F_{M}^{c}} \rightarrow \pm\left[i \omega_{n}-k_{\|} q\right] \phi_{ \pm} \overline{F_{M}^{i}}(1-b)$, where $\overline{F_{M}^{i}} \equiv \exp \left[-q^{2} v_{t i}^{2} / 2\right]$ and $v_{\mathrm{ti}}^{2} \equiv 1 / \tau$. The number densities in Eqs. (24) and (25) are nuw given by

$$
n=\bar{f}(q=0) .
$$

When the kinetic effects are not deemed important, we can use an equivalent set of fluid equations which can be obtained by taking the appropriate moments of Eqs. (19)-(22) and their ion counterparts. The same set of mode-coupling equations can also be obtained from the fluid equations in Appendix A (except for some minor differences because $b=0$ is assumed for all the nonlinear terms). These two sets of equations (kinetic and fluid) are the basis for our analytic and numerical modecoupling calculations.

In order to understand the quasilinear evolution of the instability and to identify the saturation mechanism, the mode-coupling equations are first solved analytically using a perturbative approach. The equations are manipulated into a more tractable form by exploiting the fact that the nonlinear velority-space terms are ligher order in $\epsilon$ and, therefore, cau be ignored. Furthermore, as shown in Ref. 17 , we can assume that the $(1,1)$ and $(1,-1)$ modes track each other (i.e., $\phi_{+}=\phi_{-}^{*}$ and $f_{+}=f_{-}^{*}$ ). When this is true, the $(0,2)$ terms vanish and the reduced system describes a threemode coupling process. Likewise, since the quasilinear evolution of the drift waves is dominated by the electron dynamics, only the linear density response of the fluid ions is considered. These simplifications allow us to focus on the evolution of the $(1, \pm 1)$ modes while including the effects of the $(2,0)$ mode as a perturbation. The validity of the perturbative approach as well as the consequences of these approximations will be examined later by numerical solutions of the mode-coupling equations.

As a starting point for the pertusbative analysis, let us briefly describe the collisionless case. ${ }^{17,22}$ The $\mathbf{E} \times \mathbf{B}$ convection $\left(k_{\perp}^{2} f_{0}^{c} \phi_{+}\right.$term) in Eq. (19) is the 
nonlinear perturbation, where $f_{c}^{e}$ is evaluated by $\mathrm{Eq}$. (21). Using $. Q / \partial t=-i \omega$ in the mode-coupling equations, a nonlinear dispersion relation may be derived. ${ }^{17}$ In the linear limit, it yields the ustal linear growth rate and frequency given by Eqs. (13) and (14). Nonlinearly, the real frequency of the wave is not altered, and saturation occurs when the nonlinear growth vanishes. The saturation amplitude is

$$
\left|\phi_{+}\right|_{s o}=\gamma_{l} / k_{\perp}^{2}
$$

and the quasilinear diffusion coefficient, using $\mathrm{Eq} .(17)$, for which

$$
\left\langle\Gamma_{e x}\right\rangle=\sum_{\mathbf{k}} k_{y} I m\left(\phi_{\mathbf{k}} n_{\mathbf{k}}^{e *}\right)
$$

becomes

$$
D_{\mathrm{qlo}}=\frac{2\left(1+k_{\perp}^{2}\right)^{2}}{1-b}\left(\frac{\gamma_{1}}{\omega_{*}}\right)^{2} \frac{\gamma_{l}}{k_{\perp}^{2}}
$$

The physical interpretation of the saturation is straightforward. In $k$ and $\omega$ space, the nonlinear $\mathbf{E} \times \mathbf{B}$ convection of resonant electrons eliminates the phase difference between $n_{+}^{e}$ and $\phi_{+}$thereby shutting off the growth. ${ }^{2 T}$ By constructing the Hamiltoniai of the particles in the wave frame in $x$ and $t$ space, ${ }^{29}$

$$
\begin{aligned}
& H=k_{x} k_{y} \phi \cos X \cos Y+\left(k_{\|} v_{\|}-\omega\right) X, \\
& X=k_{x} x, \quad Y=k_{y} y+k_{\|} z-\omega t,
\end{aligned}
$$

one can view the saturation as the $\mathbf{E} \times \mathbf{B}$ trapping of resonant electrons. For nonresonant particles, the $\left(k_{\|} v_{\|}-\omega\right)$ term of the Hamiltonian dominates and the phase space paths are merely straight lines. Resonant particles $\left(k_{\|} v_{\|}-\omega \simeq 0\right)$, being stationary in the wave frame, freely interact wjth the wave and contribute to the instability when $\dot{\phi} \rightarrow 0$. However, as the amplitude of the wave increases, the Iesonant particles start to move relative to the wave. Eventually, they all follow nearly circular trajectories in the phase space thereby becoming $\mathbf{E} \times \mathbf{B}$ trapped. 
When this happens, the driving mechanism of wave-particle resonance is totally ijhibited and saturation occurs.

Let us now consider the weakly collisional case where $\left|\nu_{e i} / k_{\|} v_{t e}\right| \ll 1$. For this case, it is convenient to use the Fourier-transformed Eqs. (30) and (32), for the electrons, in which nonlinear terms associated with $k_{\|} q v_{t e}^{2}$ are ignored, and $\phi_{-}^{*}=\phi_{+}, \overline{f_{-}^{e}}(q)=\overline{f_{+}^{e}}(q)$ and $\overline{f_{2}^{e}}(q)=0$. Furthermore, we neglect the collisional effects in Eq. (32). As before, with $\partial / \partial t=-i \omega$, the formal solution for $f_{+}^{e}$ assuming $k_{\|}+\nu_{\mathrm{e} i} q \neq 0$ is

$$
\begin{aligned}
\overline{f_{+}^{e}}(q) & =-\exp \left[-\int^{q} \alpha\left(q^{\prime}\right) d q\right] \int_{-\infty}^{q} \beta\left(g_{0}\right) \exp \left[\int^{q_{0}} \alpha\left(q^{\prime}\right) d g^{\prime}\right] d g_{0} \\
\alpha(q) & \equiv \frac{\nu_{e i} q^{2} v_{t e}^{2}-i \omega}{k_{\| !}+\nu_{e i} q} \\
\beta(q) & \equiv \frac{i \omega_{\cdot}+k_{\|} q v_{k_{e}}^{2}}{k_{\|}+\nu_{e i} q} \phi_{+} \overline{F_{M}^{e}}+\frac{k_{\perp}^{2}}{k_{\|}+\nu_{e q} q} \phi_{+} \overline{f_{o}^{e}}
\end{aligned}
$$

Linearly, for s.nall $\nu_{e i}$, i.e., $\left|\nu_{e i} q / k_{1}\right| \ll 1,\left|\omega_{l} / k_{i j} v_{t e}\right| \ll 1$ and $\eta_{l} \ll \omega_{l}$, we obtain

$$
\overline{f_{+}^{c}}(q)=\phi_{+}\left(\overline{F_{i j}^{c}}-i \alpha \exp \frac{i q \omega_{l}}{k_{l i}}\right)
$$

where $\alpha \equiv \sqrt{\pi / 2}\left(\ldots,-\omega_{l}\right) / k_{i j}{ }^{2 !} \ldots$. Thus, the collision frequency does not contribute to the linear stage of the drift. wave in the presence of weak collisions. By evaluating Eq. (42) at $q=0$ together with the linear ion density response,

$$
\frac{\partial n_{+}^{i}}{\partial t}+i \omega_{-} \phi_{+}(1-b)=0
$$

and Eq. (24), one derives the same expressions for $\omega_{l}$ and $\gamma_{l}$ as the collisionless case [Eqs. (13) and (14)].

Although the collisions do not greatly affect the linear stage of the instability. they can nevertheless substantially alter the nonlinear evolution of the slectron density response as given by

$$
n_{+}^{e}=\overline{f_{+}^{e}}(q=0)=\phi_{+}(1-i \alpha)-\phi_{+} \int_{-\infty}^{0} \frac{k_{\perp}^{2} \overline{f_{0}^{e}}}{k_{\|}} \exp \left[-i \frac{\omega q}{k_{\|}}+\frac{\nu_{e i} q^{3} v_{t e}^{2}}{3 k_{\|}}\right] d q \text {. }
$$


For $\overline{f_{s}^{e}}$ : let us use the quasilinear expression obtained by substituting Eq. (42) into Eq. (32),

$$
\overline{f_{0}^{e}}(q)=-i \frac{k_{\perp}^{2}}{\gamma_{l}}\left|\phi_{\perp L}\right|^{2} \alpha \exp \left[i \frac{q \omega_{l}}{k_{\|}}\right] .
$$

Along with Eqs. (24) and (43) ihe nonlinear disperstion relation becomes

$$
1+k_{\perp}^{2}-\frac{\omega_{-}}{\omega}(1-b)-i \alpha\left(1-\frac{k_{\perp}^{4} ; \phi_{+} 1^{2} I}{\gamma^{2}}\right)=0
$$

with

$$
I=\int_{0}^{\infty} \exp \left[-x-\frac{\nu_{e i} x_{\mu}^{2} v_{t e}^{2} x^{3}}{3 \gamma^{3}}\right] d x .
$$

As $\nu_{e i} \rightarrow n, I \rightarrow 1$, and the coilisionless results are recovered. However, for a nonvanishing $\nu_{c i}$, the second term in the exponent of Eq. (47) doninates, for which

$$
I \simeq \frac{\Gamma(1 / 3)}{3^{\frac{2}{3}}} \frac{\gamma_{l}}{\left(\nu_{e i} k_{\|}^{2} v_{t e}^{2}\right)^{\frac{1}{3}}}<1 .
$$

Accordingly, the nonlinear growth rate, saturation ampl:tude, and diffusion coefficient are

$$
\begin{aligned}
\gamma_{n l} & =\gamma_{l}\left(1-\frac{k_{1}^{4}}{\gamma_{i}^{2}} I\right), \\
\left|\phi_{+}\right|_{s} & =\frac{\left|\phi_{+}\right|_{s o}}{\sqrt{I}} \simeq 0.88\left|\phi_{+}\right|_{s o}\left(\frac{\nu_{e i} k_{i !}^{2} v_{t e}^{2}}{\gamma_{i}^{2}}\right)^{\frac{1}{6}}, \\
D_{q l} & =\frac{D_{q l o}}{I} \simeq 0.78 D_{q l o}\left(\frac{\nu_{e i} k_{l i}^{2} v_{t e}^{2}}{\gamma_{l}^{3}}\right)^{\frac{1}{3}}
\end{aligned}
$$

where $\left|\phi_{+}\right|_{s o}$ and $D_{\text {qlo }}$ are the collisionless saturation amplitude and diffusion coefficient given by Eqs. $(35)$ and $(36)$, respectively. Thus the saturation amplitude and resulting quasilinear diffusion may be greatly efluazced above the collisioniess values even in the presence of weak collisions.

The physical interpretation of the saturation is sinvit: $r$ to the collisionless case. In $\mathbf{k}$ and $\boldsymbol{\omega}$..space, the $\mathbf{E} \times \mathbf{B}$ convection of resonant electrons eliminates the 
phase difference between $\phi_{+}$and $n_{+}^{e}$. The notion of 'resonant particles' is still valid because we are presently considering weak collisions. In $x$ and $t$ space, as with the collisionless case, the $\mathbf{E} \times \mathbf{B}$ trapping of resonant electrons inhibits further growth of the wave. However, since collisions impair the saturation mechanism by scattering trapped particles onto nontrapping phase space trajectories, a stronger $\mathbf{E} \times \mathbf{B}$ trapping is required to overcome the destabilizing effects of the collisions. Hence, the saturation amplitude should increas sith collisions.

For the highly collisional (fluid) limit, one could use either the kinetic equations given by Eqs. (19) and (21) for the electrons or the fluid equations from Appendix A with the ordering of $\nu_{e i} \gg k_{\| l} v_{t e} \gg \omega_{l} \sim \omega_{*} \gg \gamma_{i}$ and $k_{\| l}^{2} v_{t e}^{2} \gg \nu_{e i} \omega_{l}$. We shall first use the former approach. By ignoring the nonlinear velocity-space terms and letting $\phi_{-}^{*}=\phi_{+}$, the expression for the electron distribution function becomes

$$
f_{+}^{\mathrm{e}}=\frac{\left(k_{\|} v_{\|}-\omega_{+}\right) \phi_{+} F_{M}^{e}-\zeta f_{+}^{e}+i k_{\perp}^{2} \phi_{+} f_{0}^{e}}{\left(k_{\|} v_{\|}-\omega-i \nu_{e i}\right)}
$$

where

$$
\zeta=i \nu_{e}\left[v_{i c}^{2} \frac{\partial}{\partial v_{\|}^{2}}+v_{\|} \frac{\partial}{\partial v_{\|}}-2\right]
$$

Expanding the resonant denominator in the limit of $\left(\omega+i \nu_{\mathrm{e} i}\right) / k_{\|} v_{\mid \mathrm{I}} \gg 1$, we obtain the electron density response as

$$
n_{+}^{e}=\phi_{+}\left(1-i \alpha_{c}-\frac{k_{1}^{2} \nu_{\mathrm{e} i}}{k_{\|}^{2} v_{t e}^{2}} n_{0}^{e}\right)
$$

where $\alpha_{\epsilon} \equiv \nu_{e i}\left(\omega_{*}-\omega_{l}\right) /\left(k_{\|\|} v_{t e}\right)^{2}$. In the derivation of Eq. $(54)_{2} f_{-\infty}^{\infty} d v_{\|} f_{+}^{e} v_{\beta} \sim 0$ and $f_{-\infty}^{\infty} d v_{\|}^{2} f_{+}^{e} v_{\|} \sim n_{+}^{e} v_{t e}^{2}$ are assumed. The nonlinear conrection term may be calculated by enploying the same type of perturbative approach as in the low collision limit. Substituting the linear $f_{+}^{e}\left(=f_{-}^{* *}\right)$ and $\phi_{+}\left(=\phi_{m}^{*}\right)$ in Eq. (21), and ignoring the coustribution from the velocity-space nonlinearities, we have

$$
r_{0}^{e}=-i \alpha_{c} \frac{k_{\perp}^{2}}{\gamma_{l}}\left|\phi_{+}\right|^{2} .
$$


From Eqs. (24), (43), (54), and (55), the nonlinear dispersion relation can be written as

$$
1+k_{\perp}^{2}-\frac{\omega_{*}}{\omega}(1-b)-i \alpha_{c}\left[1-\frac{k_{\perp}^{4}\left|\phi_{+}\right|^{2}}{\gamma_{l}} \frac{\nu_{e i}}{k_{\|}^{2} v_{t e}^{2}}\right]=0
$$

Linearly, it gives the results of Eqs. (13) and (15) for irequency and growth rate. As before, the nonlinear electron response has negligible effect on the real frequency. How's irer, the nonlinear growth rate becomes

$$
\gamma_{n l}=\gamma_{l}\left(1-\frac{k_{\perp}^{4}\left|\phi_{+}\right|^{2} \nu_{e i}}{\gamma_{l}\left(k_{\|} v_{l e}\right)^{2}}\right)
$$

which, in turn, gives the saturation amplitude and quasilinear aiffusion coefficient as

$$
\left|\phi_{+}\right|_{s}=\sqrt{\frac{\left(b+k_{\perp}^{2}\right)}{\left(1+k_{\perp}^{2}\right)(1-b)}} \frac{\omega_{l}}{k_{\perp}^{2}}
$$

and

$$
D_{q l}=\frac{2\left(b+k_{\perp}^{2}\right)}{1+k_{\perp}^{2}}\left(\frac{\gamma_{l}}{k_{\perp}^{2}}\right),
$$

respectively. Unlike the low collision limit where the sat.uration amplitude is strongly sensitive to $\nu_{e i}$, the saturation amplitude in the highly collisional limit is independent of collision frequency. On the other hand, the cuasilinear diffusion coefficient is proportional to the linear growth rate and, thus, increases with $\nu_{e i}$.

Nonlinear saturation of collisional drift instabilities can also be studjed using a set of fluid equations for the electrons (Appendix A). They are

$$
\begin{aligned}
& \frac{\partial n_{+}^{e}}{\partial t}+i k_{\|} u_{1-}^{e}+i \omega_{+} \phi_{+}+k_{\perp}^{2} \phi_{+} n_{-}^{k}=0 \\
& \frac{\partial n_{o}^{e}}{\partial t}=2 i k_{\perp}^{2} \operatorname{Im}\left(\phi_{+}^{*} n_{+}^{e}\right), \\
& \frac{\partial u_{\|+}^{e}}{\partial t}+i k_{\|} v_{t e}^{2}\left(n_{+}^{e}-\phi_{+}\right)=-v_{e i} u_{\|+}^{e}
\end{aligned}
$$

Letting $\partial / \partial t=-i \omega$ in Eq. (60) and neglecting the $\partial / \partial t$ term in Eq. (62), we can easily recover the nonlinear dispersion relation given by Eq. (56). 
As with the low collisional limit in $(k, w)$ space, Eqs. (54) and (55) demonstrate that the $\mathbf{E} \times \mathbf{B}$ convection term shuts off the growth of the instability by nullifying the phase difference between the density and potential response. However, the simple resonant particle saturation mechanism in $(x, t)$ space previously discussed is not applicable in the fluid limit.

In order to confirm the above quasilinear analysis, the three-mode coupling version of Eqs. (30) and (32) for both the electrons and ions including only the $\mathbf{E} \times \mathbf{B}$ electron nonlinearity and linear jon response have been numerically integrated using, the scheme presented in AF Jendix B along with Eqs. (24) and (34). The nunierjcal parameters are $\Delta q=\pi / 5 v_{t}, \pm g_{\max }= \pm 64 \Delta q$, and $\Delta t=1.0$. Figures 5 and 6 show the evolution of $e \phi(1,1) / T_{e}\left[\equiv \phi_{+}\right]$for collision rates of $\nu_{e i} / \Omega_{i}=$ $(0.0,0.001,0.01,0.1)$ for $\theta_{B}=0.01$ and 0.002 , respectively. The measured properties are $\omega / \Omega_{i}=0.046+i(0.009 \sim 0.011)$ for $\theta_{B}=0.01$ and $\omega / \Omega_{i}=(0.04-0.042)+i 0.02$ for $\theta_{B}=0.002$. They do not deviate significantly from their respective collisionless values. As predicted, collisions do not significantly affect the linear growth rate for $0 \therefore \nu_{e 1} / h_{\|} w_{t e} \simeq 1$. However, in both cases, the saturation amplitude is greatly enhanced by collisjons and appears to be rapidly approaching an upper bound as a function of $\nu_{e i}$. This trend agrees with our predictions given by Eq. (50). Most interestingly, the upper bound values coincide with the estimates of Eq. (58).

The agreement between the numerical diffusion coefficient taken at. it.s maximum value just before the saturation and the corresponding $D_{4}$ 's obtained from Eqs. (51) and (59) is not quite as good for some of the cases. The difference can be as high as a factor of $2 \sim 3$. This is due to the fact that quasilinear diffusion is really a rough estimate based purely ou linear behavior and does not take into account the nonlinear changes before saturation. Nevertheless, the trend from both calculations remains the si.iie, i.e., diffusion increases with $\nu_{e i}$. Thus, the quasilinear analysis is 
indeed adequate here.

Let us compare the three-mode coupling results with those from particle sinulation presented in Sec. III. As we can see, the saturation amplitudes indicated in Fig. 1 for $\nu_{e i} / \Omega_{i}=(0,0.001)$ and $\theta_{B}=0.01$ agree well with their corresponding values in Fig. 5. We can then conclude that nonlinear $\mathbf{E} \times \mathbf{B}$ convection for the electrons is indeed the dominant saturation mechanism for these two cases. However, the saturation levels for the other cases in Figs. 1 and 3 are considerably higher than those shown in Figs. 5 and 6, jndicating that the three-mode coupling model is not entirely valid. The quasilinear estimates for diffusion based on three-mocie coupling are also consistently lower than the simulation results. Worse yet, the model predicts zero diffusion after saturation, since there is no longer a pliase difference between density and potential. On the other hand, simulation results in Figs. 2 and 4 show nonvanishing steady-state diffusion long after saturation.

In order to understand these differences, we next. analyzed the four-mode coupling system described by Eqs. (30) to (34) for botll the electrons and ions toget.ljer with Eqs. (24) and (25), using the numerical scheme given in Appendix B. To resolve the issue of redundancy, we have discarded the dynamic equation for $\overline{f_{2}^{i}}(q=0)$ in the calculation. For the initial condition: we let $\phi_{+}=\phi_{-}$at $t=0$. Figure 6 shows the results for $\nu_{e i} / \Omega_{i}=0.001$ and $\theta_{B}=0.002$, which indicates that $\omega / \Omega_{i}=0.034+i 0.023, D_{q l} \simeq 0.02$, and $e \phi_{+} / T_{\varepsilon} \simeq 6 \%$ in the steady state. These numbers are in excellent agreement with the simulation results given in Figs. 3 and 4 . Further investigation involving the use of fluid ions ( $k_{\|}=0$ and $k_{\|} \neq 0$ ) has enabled us to identify the dominant nonlineat mechanisnus. They are the $\mathbf{E} \times \mathbf{B}$ convection for both species and the velocity-space effects for the electrons. However, most alarmingly, the four-wave coupling calculation again yields no steady-state diffusion as shown in Fig. 7, i.e., the time-averaged $\mathrm{D}$ is zero, whereas Fig. 4 gives $D=0.01$. 
Thus, the actual mechanism responsible for the nonvanishing steady-state diffusion lies elsewhere.

One possibility is that, instead of the one-dimensional diffusion madel, Eq. (23), an energy-conserving collision operator should be used in the mode-coupling calculations. Since the numerical scheme for solving the Lorentz collision operator, Eq. (3), is rather involved, we have chosen to verify this point by modifying the collision process in the simulation itself. By only using Eq. (9) for the scattering of the electrons in the code while keeping $v_{\perp j}$ unclanged, one can argue that the scheme, in fact, simulates Eq. (23). However, when this is done, the sinulation actually yields a somewhat larger diffusion coefficient in the steady state. Another possible mechanism for the particle flux in the steady state involves the non-Maxwellian feat.ure in $v_{\perp}$ for the ions due to the finite gyroradius effects which have not been included in the mode-coupling calculation. When these effects are likewise eliminated from the particle simulation code by setting $\rho_{i}=0$, there is only a slight drop ju the particle fiux for $\theta_{B}=0.01$ and no change at all for $\theta_{B}=0.002$.

By eliminating all these possibilities, we conclude that the prinary candidate giving rise to the observed steady-state diffusion in the simulation is the interaction between the background fluctuations, which have not been taken into account in the mode-coupling equations, and the dominant $\phi_{+}$and $\phi_{-}$wayes. This interaction causes the stochastic $\mathbf{E} \times \mathbf{B}$ particle transport similar to the scenario described by Horton, ${ }^{28}$ and can be understood by using the Hamiltonian of Eq. (37). Without any outside perturbations, particles in this system traverse the bounded contours of constant $\mathbf{H}$ and, as a result, there is no net particle transport. However, by adding small amplitude secondary waves to the system, particle motion near the unstable fixed points can become stochastic and results in cross-field diffusion when the isolated surfaces are destroyed. Since, in the simulation, $\phi_{+}$and $\phi_{-}$undergo 
continuous exchange of energy in the steady state due to the electron velocity-space nonlinearities, and since collisions also play an important role here, the analysis given in Ref. 28, based on a Hamiltonian of the form similar to Eq. (37), cannot be used to compare with our results quantitatively. Nevertheless, it provides us with the important insights into the origin of anomalous transport in the simulation. Further investigation of the stochastic behavior of test particles using the wave forms given by our simulation will be very helpful in understanding this process.

\section{Discussion and Conclusion}

The result.s presented in Secs. III and IV enable us to determine the relevant. mechanisms responsible for the nonlinear evolution of drift iustabilities in the presence of electron-ion collisions. While some of the nonlinearities, such as ion $\mathbf{E} \times \mathbf{B}$ convection, ${ }^{3-8}$ electron $\mathbf{E} \times \mathbf{B}$. convection ${ }^{17,20-22}$ and stochastic diffusion, ${ }^{28}$ have received considerable attention in recent years, the role of electron velocity-space nonlinearity has never been explored. The latter represents another channel for energy exchange between different modes and has significant impact on the nonlinear evolution in our simulation. By comparing the simulation results with those from mode-coupling calculations, we can also conclude that a reliable predictive model can be obtained only if one takes into account all these nonlinearities in a self-consistent manner. Such a model is, of course, not yet available, and jts development is beyond the scope of the present paper.

Despite the lack of an accurate predictive model, we can still compare our results with the trends observed in the actual experiments. ${ }^{11-13}$ Probe measurements at the tokamak edge, (where the plasma is marginally collisional) have shown that. the fluctuation levels for density and potential are rather insensitive to collisions. and particle diffusion appears to increase with local number density. In these re- 
spects, our simulation results are consistent with the experimental observations. For exaruple, the saturation amplitude in the simulation is found to be greatly increased by weak collisions and, according to the three-mode coupling model, there is an upper bound for saturation as given by Eq. (58), which is indeperdent of $\nu_{\text {ei }}$. Thus,

$$
\frac{e \phi}{T_{e}} \simeq \frac{\omega_{l}}{\Omega_{i}} \simeq \frac{\omega_{*}}{\Omega_{i}} \propto \frac{\rho_{s}}{L_{n}}
$$

for $k_{\perp} o_{s} \simeq 1$ where $L_{n} \equiv 1 /|\vec{k}|$. Such a scaling is common for many experiments. The simulation results have also indicated that particle diffusion is greatly enhanced by collisions. Since $\nu_{e i} \propto n_{e} T_{e}^{-3 / 2}$, particle diffusion in the simulation indeed increases with number density. Interestingly, quasilinear diffusion from the threemode model, $\mathrm{Eq}$. (59), also gives the same trend, i.e.,

$$
D_{q l} \simeq \frac{\gamma_{l}}{k_{\perp}^{2}} \propto n T^{-\frac{1}{2}} B^{-2}
$$

[Note that the scaling agrees with the Connor-Taylor constraint ${ }^{29}$ ] In fact, these quasilinear estimates compare favorably with the simulation results when collisions are relatively strong. For example, Figs. 2 and 4 give $D=0.004$ and 0.025 , respectively, for $\nu_{e i} / \Omega_{i}=0.01$ and $\theta_{B}=0.01$ and 0.002 , whereas $\mathrm{Eq}$. (59) gives $\mathrm{D}=0.011$ and 0.028 for the same parameters. This is rather surprising, since there is little reason based on our analysis that the two should be related. However, Horton has argued that such a correlation between stochastic diffusion and quasilinear diffusion indeed exists if there are many small amplitude waves present in the system. ${ }^{g /}$ This is certainlv the case for our simulation.

Let us now compare our results with those from recent theoretical calculations. It can be shown eastly that our simulation model in its fluid limit reduces to the one used by Hasegawa and Wakatani." Because of the way in which the k-space is truncated, the important $( \pm 2,0)$ density Auctuations lave been eliminated in their calculation. As a result, the two models predict totally different saturation 
mechanisms. Moreover, the calculation in Ref. 6 has indicated that the steadystate particle flux is linearly proportional to ion viscosity. Thus, the particle flux vanishes when ion viscosity is absent. Again, this contradicts our simulation results, in which nonvanishing steady-state diffusion exists despite the lack of ion dissipation in the sizulation plasma. This difference has been investigated by constructing conservation laws using the nonlinear gyrokinetic equations. ${ }^{30}$ The results will be reported elsewhere.

A direct comparison of our results with the calculation by Terry and Diamond ${ }^{8}$ is not feasible because of the basic difference in geometry (slab vs, toroidal) and, to a certain extent, in philosophy as well. More specifically, in their analysis, the ion energy sink was determined beforehand as the dominant saturation mechanism, whereas tluere is no prejudgment as to the relative importance of the nonlinearities included in our model. Besides, we have concentrated on nearly coherent phenomena, while their concerns were the fully developed turbulence. However, in spite of these djfferences, the scaling laws of Egs. (63) and (64), based on rather simpleminded argmments, are surprisingly similar to the ones obtained from the model in Ref. 8. The underlying reason for such a sinilarity has yet to be understood. Probably, a full-blown three-dimensional toroidal simulation has to be used to settle the issue.

In conclusion, we lave demonstrated in the present paper the usefulness of gyrolsinetic particle simulation as a computational tool for studying low-frequency microinstabilities. These studies have provided us with some highly interesting insights regarding the nonlinear behavior of drift instabilities in the presence of collisions. Based on these results, we have been able to make meaningful correlations with the existjng experimental measurements and theoretical calculations. Hopefully, our work here will stimulate further theoretical effort in understanding 
the origin and the scaling of anomalous transport. On the computational front, the next logical step is the development of the three-dimensional toroidal capability. In view of the widespread belief that the tokamak edge is of vital importance to the global confinement, such a code should prove to be extremely useful for exploring and understanding the edge physics.

\section{ACKNOWLEDGMENTS}

This research was supported by U.S. Department of Energy Contract No. DEAC02-76CHO3073. The authors gratefully acknowledge helpful discussions with Dr. W. Horton and Dr. E. J. Valeo. One of us (W. W. L.l is specially indebted to Dr. W. M. Nevins and Dr. J. A. Krommes for their valuable comments. Part of the support for J. F. F. is provided by a National Science Foundation Graduate Fellowship and by a Garden State Graduate Fellowship. 


\section{Appendix A. Fluid Equations}

A set of fluid equations associated with the gyrokinetic Vlasov-Poisson system, Eqs. (1)-( 7$)$, can be obtained by assuming that $k^{2} \lambda_{D}^{2} \ll 1, b \ll 1$, and $F=n_{o}\left(F_{M}+f\right)$, where $F_{M}$ is the spatially homogeneous Maxwellian background and is independent of time. Upon taking the appropriate velocjty moments, we arrive at

$$
\begin{aligned}
& \frac{d n^{e}}{d t}+\frac{\partial u_{\|}^{e}}{\partial x_{\|}}+\kappa \frac{\partial \phi}{\partial y}=0, \\
& \frac{d u_{\mid i}^{\epsilon}}{d t}+\frac{m_{i}}{m_{e}} \frac{\partial}{\partial x_{\|}}\left[n^{e}-\phi\right]=-\nu_{e i} u_{\|}^{e}, \\
& \frac{d n^{\prime}}{d t}+\frac{\partial u_{\mid !}^{e}}{\partial x_{\|}}+r\left(1+\frac{\nabla_{i}^{2}}{\tau}\right) \frac{\partial \phi}{\partial y}=0, \\
& \frac{d u_{\| 1}^{i}}{d t}+\frac{\partial}{\partial x_{\|}}\left[\frac{n^{i}}{\tau}+\dot{\theta}\right]=0 .
\end{aligned}
$$

and

$$
\nabla_{1}^{2} \phi=-\left(\pi^{2}-n^{\epsilon}\right)
$$

where

$$
\frac{d}{d t} \equiv \frac{\partial}{\partial t}-\frac{\partial \phi}{\partial \mathbf{x}} \times \hat{\mathbf{b}} \cdot \frac{\partial}{\partial \mathbf{x}} .
$$

In deriving Eqs. (A1)-(A6), we keep only the nonlinearities arising from $\mathbf{E} \times \mathbf{B}$ convection, for which $b=0$ is assumed. The contributions from the $\vartheta\left(\epsilon^{2}\right)$ terms in Eqs. (2) and (4) are also ignored. By letting $d u_{\|}^{e} / d t=0$ in Eq. (A2) and $\partial u_{\|}^{i} / \partial x_{\|}=0$ in Eq. (A3), and neglecting Eq. (A4) altogether, we recover the equations used in Ref. 6. 


\section{Appendix B. Integration Scheme}

The mode-coupling equations for the electrons, Eqs. (30)-(33), and the corresponding ion equations can be solved numerically using the method of characteristics. These equations assume a general characteristic form of

$$
\frac{d \bar{f}}{d t}+G(q) \bar{f}=H(q)
$$

and

$$
\frac{d q}{d t}=K(q)
$$

where $G(q) \equiv \alpha q^{2} \nu_{e i}, K(q) \equiv \beta k_{\|}+q \nu_{e i}$, and $\alpha$ and $\beta$ are constants. For

$$
\bar{f}=\bar{f} \exp \left[\int^{t} G\left(t^{\prime}\right) d t^{\prime}\right],
$$

Eq. (B1) becomes

$$
\frac{d \bar{f}}{d t}=H(t) \exp \left[\int^{t} G\left(t^{\prime}\right) d t^{\prime}\right]
$$

Thus, Eq. (B2) can be evaltzated by the numerical scheme,

$$
\bar{f}(t+\Delta t)=\left[\bar{f}(t)+H\left(t+\frac{\Delta t}{2}\right) \Delta t\right] \exp \left[-G\left(t+\frac{\Delta t}{2}\right) \Delta t\right],
$$

where $\Delta t$ is the time step. For $\mathrm{Eq}$. (B2), the solution is

$$
\begin{aligned}
q(t+\Delta T) & =q(t) \exp \left(\nu_{e i} \Delta t\right)+\frac{\beta k_{\|}}{\nu_{e i}}\left[\exp \left(\nu_{e i} \Delta T\right)-1\right] \\
& \simeq q(t) \exp \left(\nu_{e i} \Delta t\right)+\beta k_{\|} \Delta t
\end{aligned}
$$

for $\nu_{e i} \Delta t \ll 1$. There are undoubtedly better numerical methods for solving Eq. (B4). We choose this particular scheme because of its simplicity. To achieve the desired accuracy, a seven-point Lagrangian interpolation scheme has been used in the computation. 


\section{References}

${ }^{1}$ See, for example, C. M. Surko and R. E. Slusher, Science 221, 817 (1983).

${ }^{2}$ See, for example, P. C. Liewer, Nucl. Fusion 25, 543 (1985).

${ }^{3}$ A. Hasegawa and K. Mima, Phys. Rev. Lett. 39, 205 (1977); and Phys. Fluids 21, 87(1978).

${ }^{4}$ P. W. Terry and W. Horton, Phys. Fluids 26, 106 (1983).

${ }^{5}$ R. E. Waltz, Phys. Fluids 26, 169 (1983).

${ }^{6}$ A. Hasegawa and M. Wakatani, Phys. Rev. Lett. 50, 682 (1983); and M. Wakatani and A. Hasegawa, Phys. Fluids 27, 611 (1984).

${ }^{7}$ R. E. Waltz, Phys. Fluids 28, 577 (1985); and GA. Technologies Report No. GAA18204(1985).

${ }^{\star}$ P. W. Terry and I’. H. Dianıond, Phys. Flujds 28, 1419 (1985).

${ }^{9}$ W. W. Lee, Phys. Fluids 26, 556 (1983).

${ }^{10}$ D. H. E. Dubin, J, A. Krommes, C. Oberman, and W. W. Lee, Plys. Fluids 26, 3524 (1983).

${ }^{11}$ S. J. Zweben and R. W. Gould, Nucl. Fusion 23, 1625 (1983).

${ }^{12}$ S. J. Zweben and R. W. Gould, Nuci. Fusion 25, 171 (1985).

${ }^{13}$ S. J. Levinson, J. M. Beall, E. J. Powers, and R. D. Bengtson, Nucl. Fusion 24, $527(1984)$.

${ }^{14}$ W. W. Lee and H. Okuda, Phys. Rev. Lett. 36, 870 (1976); W. W. Lee, Y. Y. Kuo, and H. Okuda, Phys. Fluids 21, 617 (1978). 
${ }^{15}$ W. W. Lee, Bull. Am. Phys. Soc. 29, 1202 (1984).

${ }^{16}$ J. A. Krommes, W. W. Lee, and C. Oberman, Phys. Fluids (to be published).

${ }^{1}$ W. W. Lee, J. A. Krommes, C. Oberman, and R. Smith, Phys. Fluids 27, 2652 (1984).

${ }^{18}$ R. Shanny, J. M. Dawson, and J. M. Greene, Phys: Fluids 10, 1281 (196 $\left.\tau\right)$.

${ }^{19}$ A. Lenard and I. B. Berrstein, Phys. Rev. 112, 1456 (19.58).

${ }^{20}$ T.Sato, Phys. Fluids 17, 621 (1974).

${ }^{21}$ T. D. Rognlien and J. Weinstock, J. Geophys. Res. 79, 4733 (1974).

${ }^{22}$ R. A. Smith, J. A. Krommes, and W. W. Lee, Phys. Fluids 28, 1068 (1985).

23M. N. Rosenbluth, D. W. Ross, and D. P. Kostomarov, Nucl. Fusion 12, 3 (1972).

${ }^{24}$ R. D. Hazeltine and F. L. Hinton, Rev. Mod. Plyys. 48, 239 (1976).

${ }^{25}$ See, for example. A. H. Boozer and G. Kuo-Petravic, Phys. Fluids 24, 851 (1981).

${ }^{26}$ P. L. Bhatnagar, E. P. Gross, and M. Krooks, Phys. Rev. 94, 511 (1954).

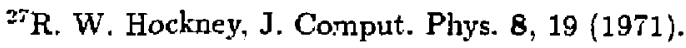

${ }^{28}$ W. Horton, Plasma Phys. 27, 937 (1985).

${ }^{29}$ J. W. Connor and J. B. Taylor, Nucl. Fusion 17, 1047 (1977).

${ }^{30}$ W. W. Lee, J. F. Federici, W. M. Täng, and W. M. Nevins, Bull. Am. Phys. Sac. 30, 1460 (1985). 


\section{Figures}

. FIG. 1. Particle simulation results for the evolution of the $\phi(1,1)$ mode for various collision rates and $\theta_{B}=0.01$.

FIG. 2. Particle simulation results for particle diffusion for various collision rates and $\theta_{B}=0.01$.

FIG. 3. Particle simulation results for the evolution of the $\phi(1,1)$ mode for various collision rates and $\theta_{B}=0.002$.

FIG. 4. Particle :jmulation results for particle riffusion for various collision rates and $\theta_{B}=0.002$.

FIG. 5. Solutions of three-mode coupling equations for $\theta_{B}=0.01$.

FIG. 6. Solutions of three-mode coupling equations for $\theta_{B}=0.002$.

FIG. 7. Time evolution for the potential and particie diffusion from four-mode coupling calculations for $\nu_{e i} / \Omega_{i}=0.001$ and $\theta_{B}=0.002$. 

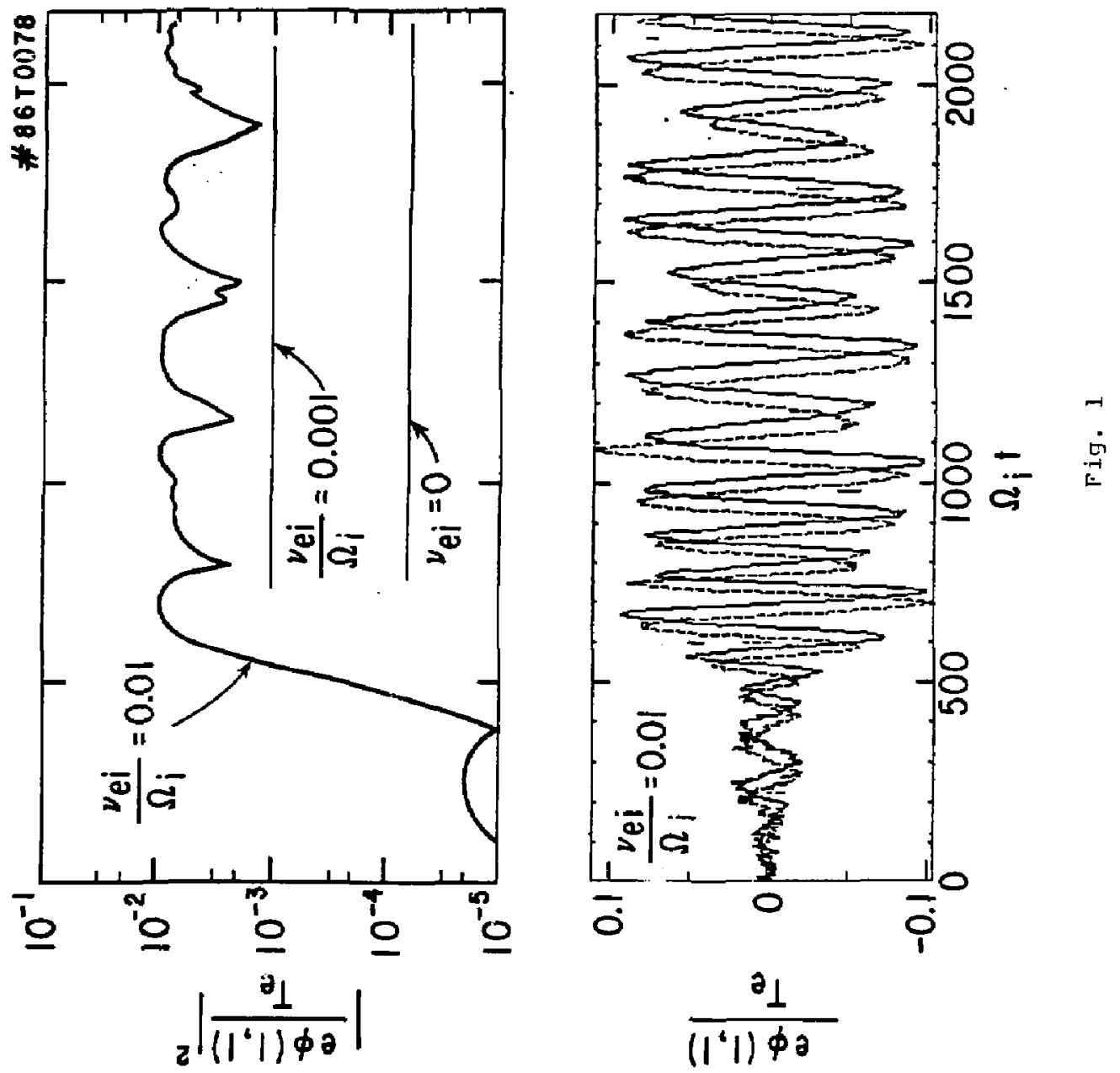


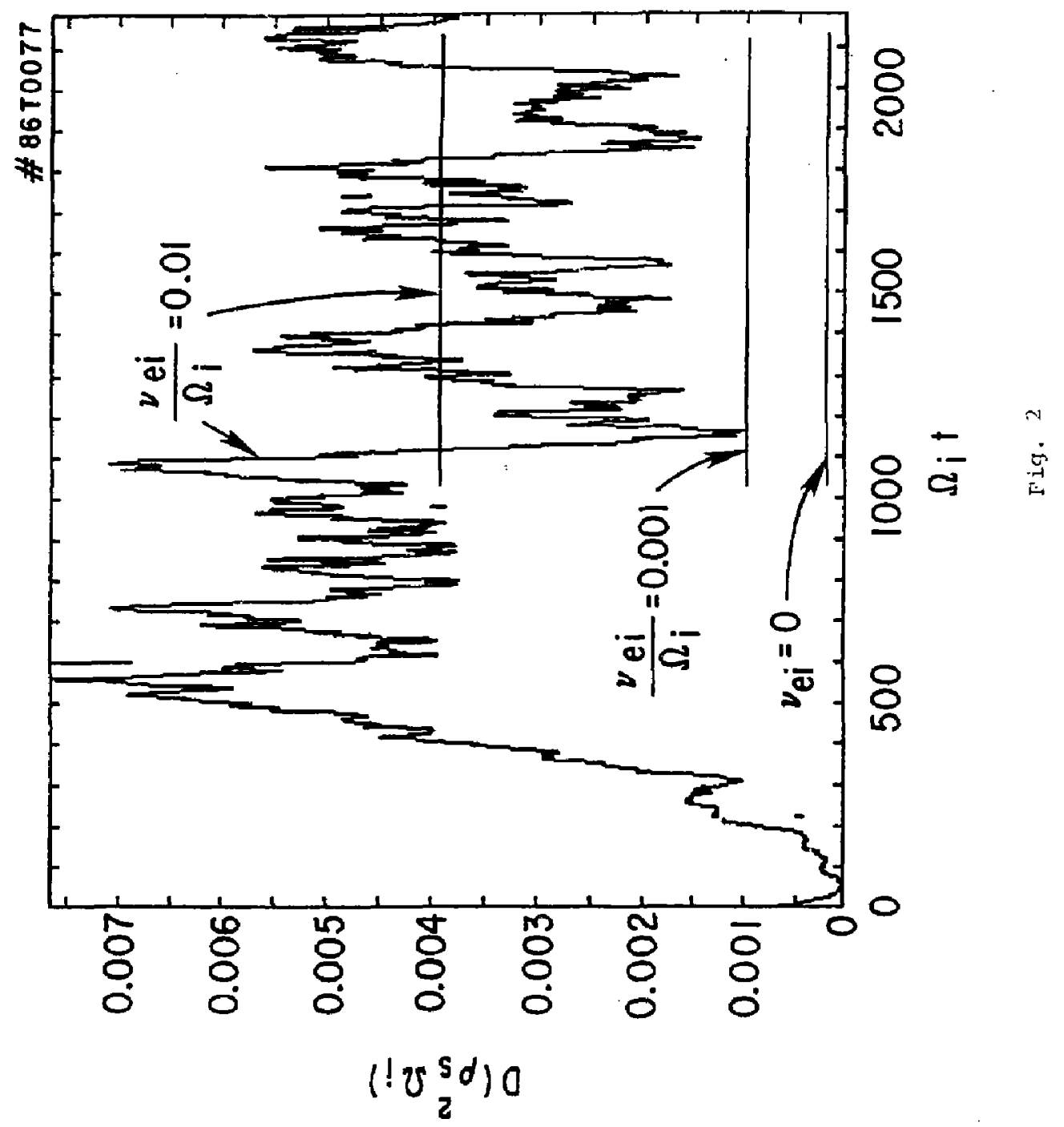



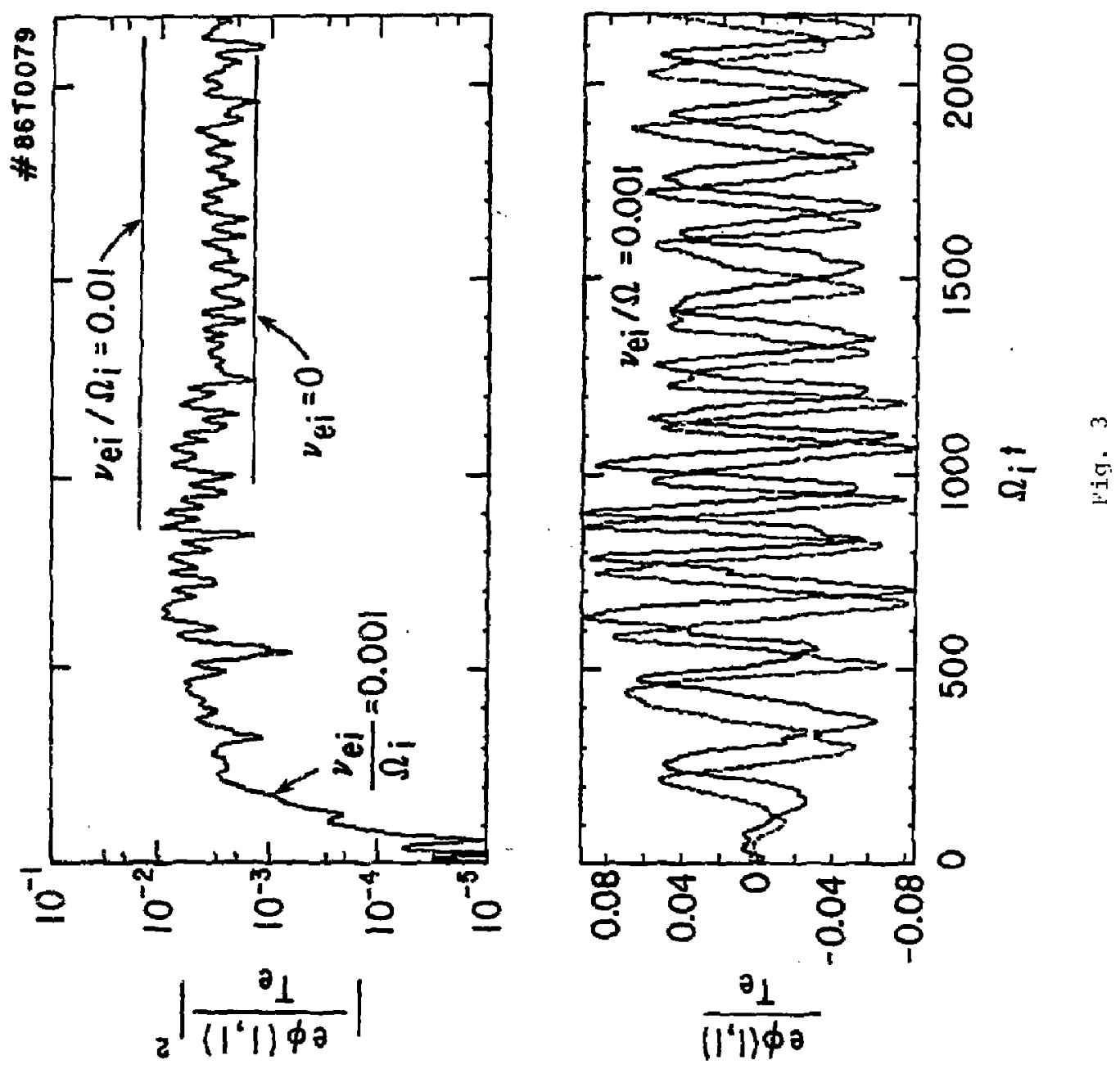

$\left|\frac{\partial_{1}}{\left\langle 1^{\prime} 1\right) \phi_{\partial}}\right|$ 


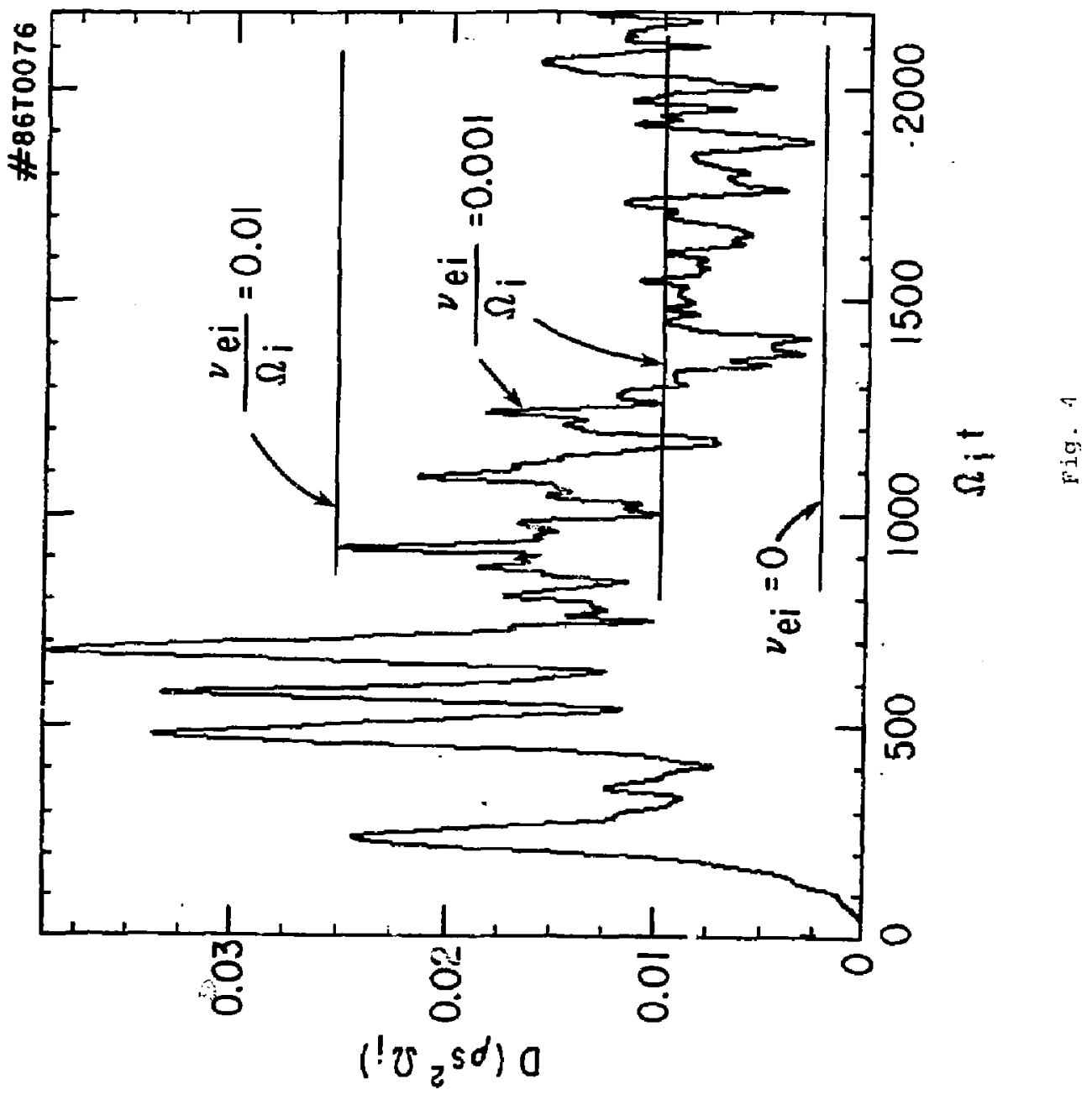



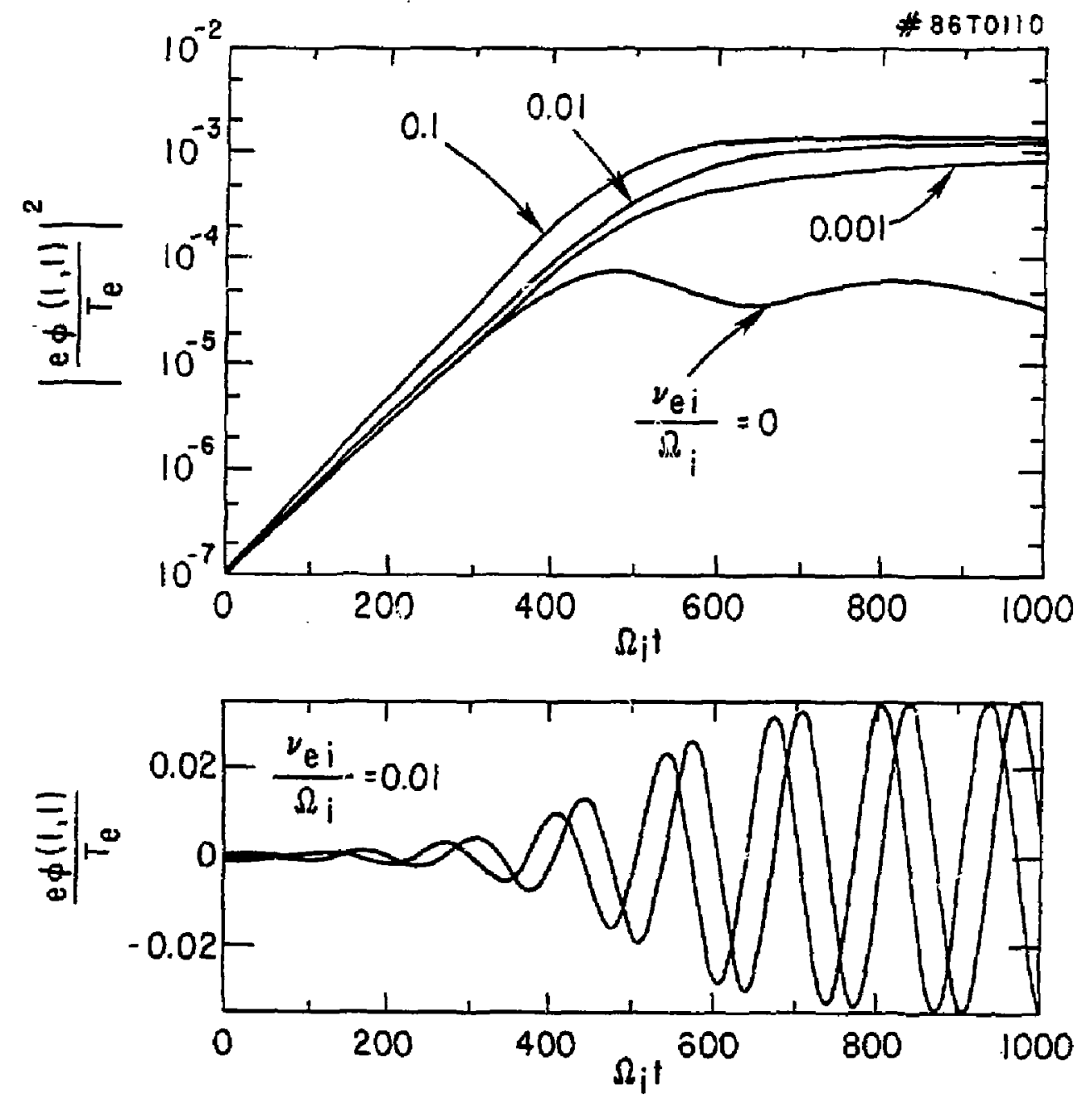

Fig. 5 

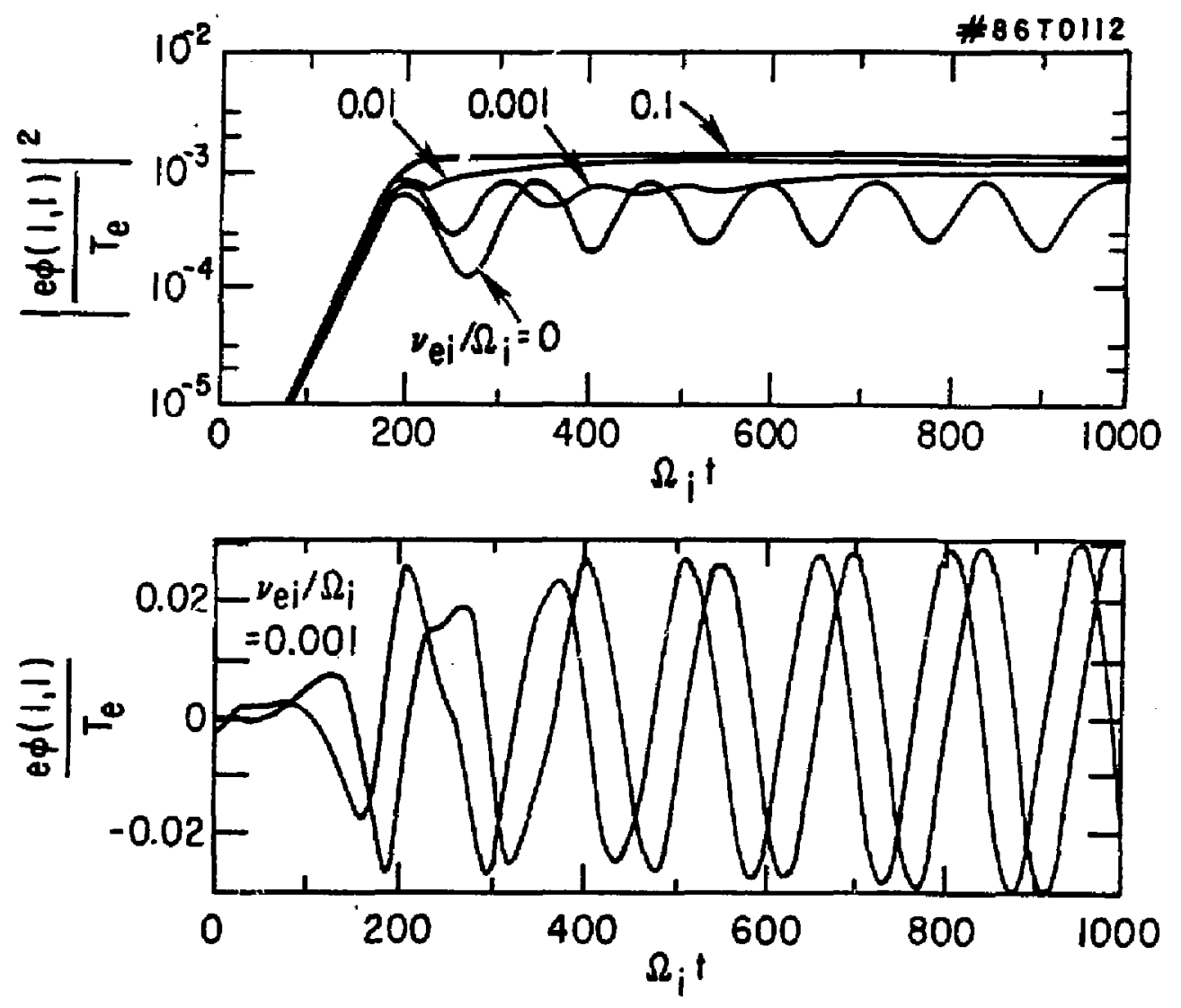

Fig. 6 

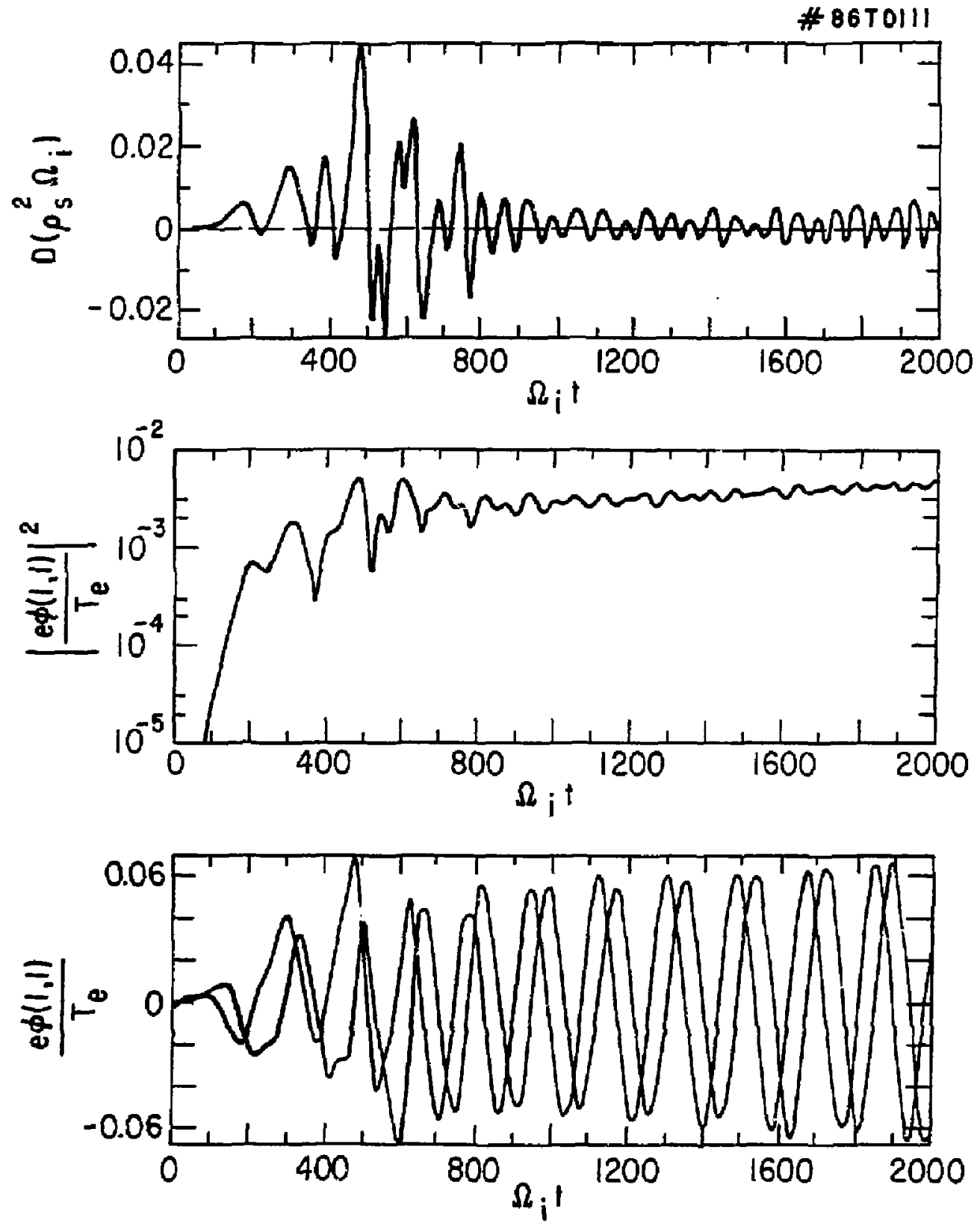

Fig. 7 
Plasma Res Lab, Austra Nar'l UnIV, AUSTRALIA

Or. Frank J. Paolonl, Unlv of ollongong, AUSTRALIA

Prof. I.R. Jones, FIIndors UnIr.. AUSTRALIA

Prot. M.H. Brennạn, Univ Sydney, AUSTRALIA

Prot. F. Cap, Inst Theo Phys, Austria

M. Gonssans, Astronomlsch InstItuut, BELGIUM

Prot. R. Bouclduo, Laboratorlion voor Natuurkunce, gELGILA

Dr. D. Palumbo, Dg XII Fuşion Prog, BELGIUM

Ecole Royale Milltalre, Lab de Phys Plasmas, aELGIUM

Dr. P.H. Sakanaka, UnIv Estadual, BRAZIL

LIb. : Doc, DIy., Instituto de Pesqulsas Espaclata, BRR IL

Dr. C.P. James, Unit of AIberta, CANAOA

Prof. J. Telchmann, UnIv of Montreal, CANADA

Or. H.M. Skarsgard, UnIV of 5oskatchewan, CANADA

Prof. S.R. Sreenlyasan, Univarslty of Calgary, CANADA

Prof. Tudor W. Johnston, INRS-Enargle, GANADA

Or. Hannes Barnard, UnIv British Columbla, CANADA

Dr. N.P. Bachynskl, MPg Tochnologies, Ine,, GANAOA

Chalk Rlyar, Nucl Lab, CANADA

Zhongwu LI, SW Inst Physles, CHINA

Library. Tsing Hua Universlty. CHINA

Llorarlan, Institute of Physles, LrINA

Inst Plosine Phys, Acadeala Sinlea, CHINA

Dr. Peter Lukac, Komanskcho UnIv, CZECHOSLOVANIA

The LIbrarlan, Culthan Laboratory. ENGLAND

Prof. Schatzmon, Observotolre de NICe, FRANCE

J. Rindet, CEN-GP6, FRANCE

JET Rasdirg Room, JET Jolnt Undertaking. ENGLAND

M Dupas Library, M Dupos Library, FRANCE

Dr. Tom Hual, Acadeny Bi billographIc, HONG KONG

Proprint LIbrary, Cent Res Inst Phys, HUNGARY

Dr. R, K, Chhajtant, VIkram Unlv, INDIA

Dr. B, Er jupta, Saha Inst, INOIA

Dr. P. Kaw, Physical Research Lab, InOIA

Dr. PhIlllid Rosenou, Isroel Inst Toch, ISRAEL

Prof. S. Cuperman, Tal Avlv University, ISRAEL

Prof. G. Rastagni, Univ DI Padova, ITALY

Librarian, Int'I Ctr Theo Phys, ITALY

miss Clolía De Palo, Assoc ElLATOM-ENEA, ITALY

Bibllateca, del CNR ELRATUA, ITALY

Dr. H. Yawato, ToshIbs Res Dov, JAPAN

Direc. Dapt. Lg. Tokomok Dew. JAERI, JAPAN

Prot. Hobuyukl Inowe, Unlversity of Tokyo, JAPAN

Research Info Center, Nagyo UnIvorsity, MAPAN

Prof. Kyoji NIshIkawa, UnIV of HIroshIno, JAPAN

Prof. SIgeru MorI, JAERI, JAPAN

Prot. 5, Tanaka, Kyoto University, JAPAN

LI berify, Kyoto University, JAPAN

Prof. Ichlro Kawakaml, N1hon UnIV, JAPAN

Prof. Satoshl Iton, Kyushu University, JAPAN

Dr. D, I, Chol, Adv. Inst Sel \& Tech, KOREA

Toch Into Division, KAERI, KOREA
Bibllotheck, Fom-Inst Voor Plasmo, NeTHealanos

Prot. B.S. Lilly, Unl versity of Watkato, NEW ZEMLAND

Prot. J.A.G. Gabral, Inst Superfor Teen, POATUGal

Dr. Octavien Petrus, ALI CleA Unlversity, ROManiA

Prot. H.A. Helloerg, Unlverslty of Natal, SO AfRICA

Dr. Johan de Villlers, Plasin Physics, Nucor, SO AFRICA

Fuslon Div. LIorary, JEN, SPANN

Prot, Hans Wlihelmson, Chalmers Unlv Tech, SMEDEN

Dr. Lennart Stenflo, Unlversity of UMEA, SWEDEN

LIbrary. Royal Inst Twch, SWEDEN

Centre do Recherchesen, Ecole Polytech Fod, SWITEERLAND

Dr. V.T. Tolok, Kharkoy Phys Toch Ins, USSR

Dr. 0.0. Ryutov, SI berian Acad Sel. USSR

Dr. G.A. Ellseov, Kurchatov Institute, US5R

Dr. V.A. Glukhikh, Inst Eloctrofhysleal, USSR

Institute Gen. Physics, USSR

Praf. T.J.M, Bayd, Univ College N Wales, MALES

Or. K. Schindler, Ruhr Unlvarsitat, W. GERMANY

ASDEX Raading Rm, IPPMax+lanck-l nstitut fur

Plasmaphysik, F.R.G.

Nuclear Ras Estob, Jullich Ltd, W. GERMANY

LIbrarlan, Maxtilanck Institut, $H_{*}$ GERMANY

Blbltathek, Inst Plasmaforschung, W. GERMANY

Prot. R.K. Janev, Inst Phys, YueOSLIVIA 